\title{
A Probabilistic Paraconsistent Logical Model for Non-Relativistic Quantum Mechanics Using Interlaced Bilattices with Conflation and Bernoulli Distribution
}

\author{
João Inácio Da Silva Filho \\ Laboratory of Applied Paraconsistent Logic, Santa Cecília University—UNISANTA, Santos, Brazil \\ Email: inacio@unisanta.br
}

How to cite this paper: Da Silva Filho, J.I. (2017) A Probabilistic Paraconsistent Logical Model for Non-Relativistic Quantum Mechanics Using Interlaced Bilattices with Conflation and Bernoulli Distribution. Journal of Quantum Information Science, 7, 89-124.

https://doi.org/10.4236/jqis.2017.73009

Received: August 29, 2017

Accepted: September 25, 2017

Published: September 28, 2017

Copyright $\odot 2017$ by author and Scientific Research Publishing Inc. This work is licensed under the Creative Commons Attribution International License (CC BY 4.0).

http://creativecommons.org/licenses/by/4.0/

\begin{abstract}
In this work, we make a representation of non-relativistic quantum theory based on foundations of paraconsistent annotated logic (PAL), a propositional and evidential logic with an associated lattice FOUR. We use the PAL version with annotation of two values (PAL2v), named paraquantum logic (PQL), where the evidence signals are normalized values and the intensities of the inconsistencies are represented by degrees of contradiction. Quantum mechanics is represented through mapping on the interlaced bilattices where this logical formalization allows annotation of two values in the format of degrees of evidence of probability. The Bernoulli probability distribution is used to establish probabilistic logical states that identify the superposition of states and quantum entanglement with the equations and determine the state vectors located inside the interlaced Bilattice. In the proposed logical probabilistic paraquantum logic model ( $p$ PQL Model), we introduce the operation of logical conflation into interlaced bilattice. We verify that in the $p$ PQL Model, the operation of logical conflation is responsible for providing a suitable model for various phenomena of quantum mechanics, mainly the quantum entanglement. The results obtained from the entanglement equations demonstrate the formalization and completeness of paraquantum logic that allows for interpretations of similar phenomena of quantum mechanics, including EPR paradox and the wave-particle theory.
\end{abstract}

\section{Keywords}

Paraconsistent Logic, Interlaced Bilattice, Probability Theory, Quantum Mechanics, Paraquantum Logic Model 


\section{Introduction}

Studying the effects of undulatory theory of light, researchers found that experiments in modern physics dealing with quantum mechanics lead to results that are incompatible with classical physics. This contradiction between classical theory and modern experimental research requires a fundamental modification of basic physical concepts and laws so that they can apply to atomic phenomena [1] [2] [3] [4]. From these observations, investigations have been conducted to find the foundations of a new logic more suitable for creating equations and models for quantum mechanics [5].

The formalization of quantum logic has been studied in various ways; the best known of these was first presented in 1936 by John von Neumann and Garret Birkhoff [5], in which the authors discussed a new form of logic which, due to the quantum phenomena observed, would be incompatible with classical logic [5] [6]. Other authors studied formalization of a quantum logic able to support logical models that could answer the questions relating to quantum phenomena [7]-[15]. Among these works, we highlight those [13] [14] [15] where the authors use non-classical logics such as fuzzy and paraconsistent logic to create the formalization of a quantum logic.

The probabilistic logical model presented in this work uses all the established concepts of quantum mechanics with foundations of the non-classical logic such as paraconsistent annotated logic (PAL) with the annotation of two values (PAL2v) [16]-[23], the interlaced bilattices [6] [24] [25] [26] [27], Bernoulli distribution, and probability theory [28] [29].

This paper is organized as follows: in section 2, we present some of the key concepts of quantum mechanics, probability theory, and Bernoulli distribution. In section 3, we present some concepts of non-classical paraconsistent logics and some representations by lattices, highlighting the main concepts of PAL. In section 4, we present the bilattices theory and the algebra of interlaced lattices. In section 5, we present the paraquantum logic (PQL) equations of the evidence and degrees of certainty and those of the contradiction represented in Belnap's bilattices. At the end of this section, we combine PQL with the theory of Bernoulli trials and the probability calculations, variance, and standard deviation. In Section 6, we present the results obtained by interpretations of the probabilistic paraquantum model and discuss their fundamentals and concepts. At the end of this section, we discuss the EPR paradox with the interpretation of the probabilistic paraquantum model and an application example. In section 7 , we present our conclusions.

\section{Concepts of Quantum Mechanics (QM)}

QM, a branch of physics, studies the fundamental theory of nature at the small scales and energy levels of atoms and subatomic particles. In the mathematical formulation of QM, the state of a system at any given time is described by a complex wave function, also referred to as a state vector in a complex vector space [3] 
[11]. This abstract mathematical object enables the calculation of probabilities for the outcomes of actual experiments [1] [3] [4] [7] [9] [11] [13] [14] [15].

In contrast to classical mechanics, in quantum mechanics, any unit vector, which is a linear combination of pure states, gives rise to a new pure state. Because two pure states $\psi_{1}$ and $\psi_{2}$ are orthogonal and assuming that a pure state $\psi$ is a linear combination of $\psi_{1}$ and $\psi_{2}$, we can write as follows [2] [5] [12]:

$$
\psi=c_{1} \psi_{1}+c_{2} \psi_{2}
$$

where $\left|c_{1}\right|^{2}+\left|c_{2}\right|^{2}=1$

For a quantum system in state $\psi$, those events (and experimental propositions) that are certain for state $\psi_{1}$ but not for $\psi_{2}$ are verified with probability $\left|c_{1}\right|^{2}$ and those events (and experimental propositions) that are certain for state $\psi_{2}$ but not for $\psi_{1}$ are verified with probability $\left|c_{2}\right|^{2}$ [2] [5] [30].

\subsection{Superposition and Hilbert Space}

Superposition appears in the study of classical physics as well as in quantum physics. The superposition principle in the current formulation of quantum theory is given precise mathematical meaning through the Hilbert space formalism. The principle of superposition of states affirms that the complex linear superpositions of Equation (1) also represent the quantum states of the system [1] [7] [9].

\subsection{Quantum Logic and Hilbert Space}

Quantum logic is defined as the logic of Orthomodular lattices (OMLs) [5,6] and is conceptually very similar to the inherent properties of Hilbert space. The OML was introduced in 1936 by Birkhoff and von Neumann as an algebraic account of the logic of quantum mechanics [5] [6] [9]-[15].

\subsection{Entanglement in Quantum Mechanics}

One of the main phenomena of QM is the entanglement, considered to be the most non-classical manifestation of quantum formalism. The quantum entanglement is a quantum mechanical property that allows two or more objects to be related in such a way that it is not possible to describe one of them completely without relating it to the other(s). Entanglement can be studied from the initial condition that a pure quantum state can be represented by a vector in a complex Hilbert space with unit length. Thus, for each pure state $|\psi\rangle$ and any basis $\left\{\left|u_{1}\right\rangle, \cdots,\left|u_{n}\right\rangle\right\}$, the state $|\psi\rangle$ can be extended to $|\psi\rangle=\alpha_{1}\left|\psi_{1}\right\rangle+\cdots+\alpha_{n}\left|\psi_{n}\right\rangle$, where $\sum_{i=1}^{n}\left|\alpha_{i}\right|^{2}=1$. A pure state $\left|\psi_{A 1, A 2, \cdots, A n}\right\rangle$ with $m$ subsystems is completely separable into parts only if it can be written as $\left|\psi_{A 1, A 2, \cdots, A n}\right\rangle=\left|\psi_{A 1}\right\rangle\left|\psi_{A 2}\right\rangle \cdots\left|\psi_{n}\right\rangle$. If it cannot be written in this way, then the state is said to be entangled [7] [10] [11].

\subsection{Observables and Measurement in Quantum Mechanics}

In a general way, in Physics, an observable $\mathrm{A}$ is considered a dynamic variable that can be measured. For example, the position and momentum of a particle are 
considered observables [7] [10] [11]. In quantum physics, the interpretation is different, and an observable $\mathrm{A}$ is an operator or a gauge, where the property of the system state can be determined by some sequence of physical operations that may involve submitting the system to various electromagnetic fields and obtaining a value [8] [10]. Physically meaningful observables must also satisfy transformation laws that relate to observations made by different observers in different frames of reference.

\subsection{Probability in Quantum Mechanics}

The principles of QM that define the probability $p$ of an event occurring is given by the square of the norm of a complex number $\alpha$ (where $\alpha$ is called probability amplitude or magnitude) [2] [3] [4] [5] [7] [12] [30] [31].

$$
p=\|\alpha\|^{2}
$$

The probability amplitude $\alpha$ is an event that will be denoted by

$$
\alpha=\langle\text { final state }| \text { initial state }\rangle
$$

As can be seen in [15] and [16] [31], there are difficulties with a rigorous definition of probability for finding a better understanding of applications in QM. For assigns to an event $X$, probability $=1(0$, respectively) if and only if assigns to the orthocomplement of $X$ probability $=0$ (1, respectively). Consequently, we are dealing with an operation that inverts the two extreme probability values, which naturally correspond to the truth-values truth and falsity (as in the classical truth table of negation) [2] [5] [7] [8].

\subsection{Bernoulli Trial Process}

The process of Bernoulli trials, named after Jacob Bernoulli, is one of the simplest random processes in probability, but very important in QM. Due to the probabilistic nature of $\mathrm{QM}$, the Bernoulli trial process is useful for studying statistical quantum phenomena [28] [29] [32]. Basically, the Bernoulli trial process is the mathematical abstraction of coin-tossing, and because of its wide applicability, it is usually stated in terms of a sequence of generic trials. A sequence of Bernoulli trials must satisfy the following assumptions:

1) Each trial has two possible outcomes called success $(k=1)$ and failure $(k=0)$.

2) The trials are independent; the outcome of one trial has no influence over the outcome of another trial.

3) In each trial, the probability of success is $p$ and the probability of failure is 1 $-p$, where $p \in[0,1]$ is the success parameter of the process.

\subsection{Probability Mass Function ( $p m f$ ) in the Bernoulli Trial Process}

Let $p$ be the probability of success in a Bernoulli trial, and $q$ be the probability of failure, then the probability of success and the probability of failure sum to unity (one). Since these are complementary events, success $(p)$ and failure $(q)$ are mutually exclusive and exhaustive [28] [32]. 
The probability measure $p$ is a function that relays an event's probability. We can consider that impossible events have a probability zero, and the probability is one if the event is certain to happen. Thus, $p$ is a function $p: F \rightarrow[0,1]$. The probability measuring function must satisfy the simple requirement that the probability of a countable union of mutually exclusive events is equal to the countable sum of the probabilities of each of these events [32].

The probability mass function $(p m f)$ of this distribution, over possible outcomes $k$, is

$$
f(k ; p)=p^{k}(1-p)^{1-k} \text { for } k \in\{0,1\}
$$

\subsection{Expectation Value in the Bernoulli Trial Process}

The Bernoulli trial process for a distributed random variable $X$ is $\operatorname{Pr}(X=1)=p$ and $\operatorname{Pr}(X=0)=q$. The expectation value is

$$
\begin{array}{r}
E(X)=\operatorname{Pr}(X=1) \times 1+\operatorname{Pr}(X=0) \times 0 \\
E(X)=p \times 1+q \times 0 \\
E(X)=p
\end{array}
$$

\subsection{Variance $\left(\operatorname{Var}(X)=\sigma^{2}\right)$ and Standard Deviation $(\sigma)$ in the Bernoulli Trial Process}

The variance of $X$, written as $\operatorname{Var}(X)$ or indicated by the symbol $\sigma^{2}$, is a measure of how much the value of $X$ varies from the expectation $E(X)$ [32]. $\operatorname{Var}(X)$ has a central role in statistics and is defined as the expectation of the squared deviation of a random variable from its mean. In the Bernoulli distribution, this is defined as follows:

Let $X$ be a discrete random variable with the Bernoulli distribution and probability parameter $p$. Since the variance is the weighted sum of the squared distances from the mean it involves the probability for which we get 0 and the probability for which we get 1 . The variance of $X$ is then given by [32]

$$
\begin{aligned}
\operatorname{Var}(X)=\sigma^{2} & \rightarrow \sigma^{2}=\left[(1-p)(0-p)^{2}\right]+\left[p(1-p)^{2}\right] \\
& \rightarrow \sigma^{2}=(1-p)(p)^{2}+p\left(1-2 p+p^{2}\right) \\
& \rightarrow \sigma^{2}=(1-p)(p)^{2}+p 2 p^{2}+p^{3} \\
& \rightarrow \sigma^{2}=p^{2}-p^{3}+p-2 p^{2}+p^{3} \\
& \rightarrow \sigma^{2}=p-p^{2}
\end{aligned}
$$

The standard deviation of a probability distribution is given by symbol $\sigma$ and is defined as the square root of the variance $\sigma^{2}$ :

$$
\sigma=\sqrt{\sigma^{2}}
$$

\subsection{EPR Paradox}

In 1935 the paper [33] brought a text originally proposed to exhibit internal 
contradictions in the new quantum physics. The text is a paradox and it was resulted of thought experiment proposed by Albert Einstein, Boris Podolsky, and Nathan Rosen. Known today as the EPR paradox the authors hoped to show that quantum theory could not describe certain intuitive "elements of reality" and thus was either incomplete or demonstrably incorrect.

\section{Non-Classical Paraconsistent Annotated Logic}

A formal system based on logic binary principles that differs in a significant way from classic logical systems is considered a non-classic logical system.

There are several types of non-classical logics and the aim of this formalization is to construct different models of logical consequence and logical truth [15] [17] [25]. A paraconsistent logic is a non-classical logic whose main foundation is its tolerance to contradiction without trivialization [14] [15].

\subsection{Paraconsistent Logic and Many-Valued Logic}

Many-valued logic is a non-classical logic because it rejects bivalence, allowing for truth values other than true and false and can be represented by lattices [25] [27]. With these fundamentals, the many-valued logics present characteristics of the paraconsistent logics family.

\subsection{Paraconsistent Four-Valued Logic}

Belnap [5] [24] provides a semantic characterization of a complex four-valued logic that aims to formalize the internal states of a computer.

An appropriate non-classical axiomatization is defined that captures the semantic where there are four states: $(t),(f),(N)$, and $(B)$, where $(N)$ and $(B)$ are abbreviations of (None) and (Both), respectively.

Based on these four states recognized as input, a computer can determine the suitable outputs [24] [26]. Belnap's four-valued logic is paraconsistent logic and can model both incomplete (N) and inconsistent (B) information [5] [24].

In propositional logic concepts, we have the following:

$(t)$ the proposition is true

$(f)$ the proposition is false

$(N)$ the proposition is neither true nor false

$(B)$ the proposition is both true and false

From this, $(N)$ corresponds to incompleteness and $(B)$ inconsistency.

Four-valued logic has logical symbols $\sim, \wedge, \vee$ and is based on the approximation lattice with a different ordering.

In Belnap's work [5] [24], semantics for the language of four-valued logic with the logical symbols are presented.

\subsection{Paraconsistent Annotated Logic (PAL)}

PAL [19] [20] [21] is an extension of paraconsistent logic and it can be presented with the annotation of two values (PAL2v). 
In the PAL2v representation, an atomic proposition of logic language PAL can be presented by $p[\mu, \lambda]$, where $p$ denotes a proposition or propositional variable; the truth value (or annotation with two values) $[\mu, \lambda] \in \operatorname{set}[0,1] \times[0,1]$; and the values $\mu, \lambda$ are elements in a closed interval $[0,1]$ of a set of real numbers [17] [18] [19] [20] [21].

An order relation is defined on $[0,1]^{2}:\left[\mu_{1}, \lambda_{1}\right] \leq\left[\mu_{2}, \lambda_{2}\right] \Leftrightarrow \mu_{1} \leq \mu_{2}$ and $\lambda_{1} \leq \lambda_{2}$, constituting a lattice that will be symbolized by $\tau$. The operator $\neg:|\tau| \rightarrow|\tau|$ defined the logical negation in the lattice, as

$$
\neg[\mu, \lambda]=[\lambda, \mu]
$$

where $\mu, \lambda \in[1,0] \subset \Re$

We note that $\tau$ is a complete lattice under $\leq$. The bottom element of the lattice $\tau$ is $[0,0]$, the paracompleteness state, whose symbol is $\perp$, and the top element is the inconsistent state $[1,1]$, whose symbol is $\mathrm{T}$. Therefore, the PAL has an associated lattice $\tau$ (lattice FOUR), as shown in Figure 1, in which the connotation of logical states can be assigned to its vertices [18] [20] [21].

\section{Theory of Bilattices}

Bilattices are algebraic structures introduced in 1988 by Ginsberg [34] as a uniform framework for inference and logical analysis in the field of artificial intelligence. These bilattice structures were presented in order to formalize hypothetical and uncertain reasoning and were extensively studied by Ginsberg and others involved in programming logic and truth theory [20] [34].

\subsection{Bilattice Representation}

In general, a bilattice representation has two kinds of ordering, a truth ordering $\leq_{t}$ and a knowledge ordering $\leq_{k}$. Therefore, a bilattice is a structure $B=\left\langle\mathrm{B}, \leq_{\mathrm{t}}, \leq_{k}, \neg\right\rangle$, where $\mathrm{B}$ denotes a non-empty set and $\leq_{\mathrm{t}}$ and $\leq_{k}$ are partial orderings on $B$ [20] [25] [34].

The ordering $\leq_{k}$ is described as ranking the "degree of information (or knowledge)".

The bottom in $\leq_{k}$ is denoted by $\perp$-Paracomplete and the top by T-In-

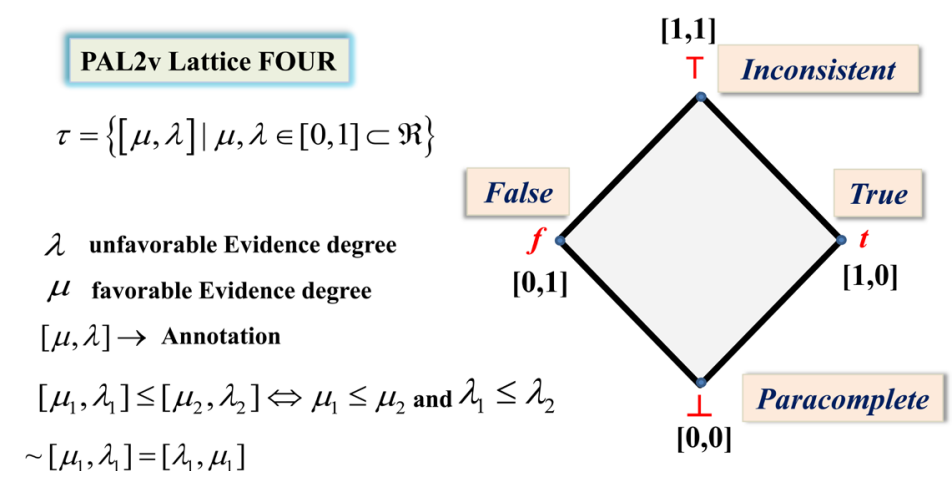

Figure 1. Paraconsistent annotated logic with annotation of two values (PAL2v) and associated lattice FOUR with annotations and logical conotation in its vertices. 
consistent.

The ordering $\leq_{t}$ is describing as ranking the "degree of truth".

The bottom in $\leq_{t}$ is denoted by $f$-false and the top by $t$-true.

A bilattice $B$ is defined by Ginsberg [34] as a sextuple $B=\langle\mathrm{B}, \wedge, \vee, \otimes, \oplus, \neg\rangle$ such that:

1) The $t$-Lattice $\left(\mathrm{B}, \leq_{t}, \vee, \wedge\right)$ and the $k$-Lattice $\left(\mathrm{B}, \leq_{k}, \otimes, \oplus\right)$ are both complete lattices.

2) $\neg: B \rightarrow B$ is an involution ( $\neg \neg$ is the identity) mapping such that $\neg$ is lattice homomorphism from $(B, \vee, \wedge)$ to $(B, \wedge, \vee)$ and $(B, \otimes, \oplus)$ to itself [20] [34].

The two operations corresponding to this ordering ( $t$-Lattice) are the meet (greatest lower bound) $\wedge$ and the join (least upper bound) $\vee$. The operations $\otimes$ and $\oplus$ correspond to the greatest lower bound and the least upper bound, respectively, in the knowledge ordering ( $k$-Lattice).

The minimum and maximum elements of the lattice $\langle B, \wedge, \vee\rangle$, if they exist, will be denoted, respectively, by $f$ and $t$ [20] [25] [34].

\subsection{Interlaced Bilattice}

An interlaced bilattice is a structure $B=\left\langle B, \leq_{t}, \leq_{k}\right\rangle$, where

1) both $\leq_{t}$ and $\leq_{k}$ give $B$ the structure of a lattice and

2) the meet and join operations for each partial ordering are monotone with respect to the other ordering: $x \leq_{t} y \rightarrow x \oplus z \leq_{t} y \oplus z$ and $x \otimes z \leq_{t} y \otimes z$

$$
x \leq_{k} y \rightarrow x \vee z \leq_{\mathrm{t}} y \vee z \text { and } x \wedge z \leq_{\mathrm{t}} y \wedge z
$$

Bilattices are required to be complete, and they have tops and bottoms. Interlaced bilattices, as defined above, do not have a completeness requirement, but it is assumed that all such structures have tops and bottoms with respect to both orderings [20] [25] [34]. In an interlaced bilattice, an operation associated with one of the lattice orderings is required to be monotonic with respect to the other lattice ordering. Note that this condition is a different kind of connection between the two orderings considered via negation.

In general, the interlaced bilattice properties can be defined as follows:

Definition 1 - Let $\left\langle\mathrm{L}_{1}, \leq_{1}\right\rangle$ and $\left\langle\mathrm{L}_{2}, \leq_{2}\right\rangle$ be lattices.

By $\mathrm{L}_{1} \circ \mathrm{L}_{2}$, we mean the structure $\left\langle\mathrm{L}_{1} \times \mathrm{L}_{2}, \leq_{\mathrm{t}}, \leq_{k}, \neg\right\rangle$, where

1) $\left\langle x_{1}, x_{2}\right\rangle \leq_{\mathrm{t}}\left\langle y_{1}, y_{2}\right\rangle$ provides $\left\langle x_{1} \leq_{1} y_{1}\right\rangle$ and $\left\langle y_{2} \leq_{2} x_{2}\right\rangle$ and

2) $\left\langle x_{1}, x_{2}\right\rangle \leq_{k}\left\langle y_{1}, y_{2}\right\rangle$ provides $\left\langle x_{1} \leq_{1} y_{1}\right\rangle$ and $\left\langle x_{2} \leq_{2} y_{2}\right\rangle$.

If $\mathrm{L}_{1}$ and $\mathrm{L}_{2}$ are lattices (with tops and bottoms), $\mathrm{L}_{1} \circ \mathrm{L}_{2}$ is an interlaced bilattice.

Furthermore, if $\mathrm{L}_{1}=\mathrm{L}_{2}$, then the operation given by $\neg\langle x, y\rangle=\langle y, x\rangle$ satisfies the negation conditions of definition 1 of bilattices.

Finally, if both $\mathrm{L}_{1}$ and $\mathrm{L}_{2}$ are complete lattices, $\mathrm{L}_{1} \circ \mathrm{L}_{2}$ satisfies the completeness condition for bilattices.

By intuition, we consider that if $(x ; y) \in \mathrm{L}_{1} \times \mathrm{L}_{2}$, then $x$ represents the information for some assertion, and $y$ is the information against it. 
Other properties that permit logical operation of $\mathrm{L}_{1} \circ \mathrm{L}_{2}$ are given below.

- Let $\mathrm{L}$ be a complete lattice with a join $\cup_{\mathrm{L}}$ and a meet $\cap_{\mathrm{L}}$. Then $\mathrm{L}_{1} \circ \mathrm{L}_{2}$ is a bilattice with the following basic operations:

$$
\begin{gathered}
\left(x_{1}, y_{1}\right) \vee\left(x_{2}, y_{2}\right)=\left(x_{1} \cup_{\mathrm{L}} x_{2}, y_{1} \cap_{\mathrm{L}} y_{2}\right) \\
\left(x_{1}, y_{1}\right) \wedge\left(x_{2}, y_{2}\right)=\left(x_{1} \cap_{\mathrm{L}} x_{2}, y_{1} \cup_{\mathrm{L}} y_{2}\right) \\
\left(x_{1}, y_{1}\right) \oplus\left(x_{2}, y_{2}\right)=\left(x_{1} \cup_{\mathrm{L}} x_{2}, y_{1} \cup_{\mathrm{L}} y_{2}\right) \\
\left(x_{1}, y_{1}\right) \otimes\left(x_{2}, y_{2}\right)=\left(x_{1} \cap_{\mathrm{L}} x_{2}, y_{1} \cap_{\mathrm{L}} y_{2}\right) \\
\neg\left(x_{1}, y_{1}\right)=\left(y_{1}, x_{1}\right) \\
\neg\left(x_{2}, y_{2}\right)=\left(y_{2}, x_{2}\right)
\end{gathered}
$$

In an interlaced bilattice, we take a conflation $(-)$ of $T$ to be $\perp$, and conversely, negation $(\neg)$ of true $t$ and false $f$ to be themselves again. Then lemma:

For logical negation,

$$
\begin{aligned}
\neg\left(x_{1} \wedge y_{1}\right)=\neg x_{1} \vee \neg y_{1}, & \neg\left(x_{1} \vee y_{1}\right)=\neg x_{1} \wedge \neg y_{1} \\
\neg\left(x_{1} \otimes y_{1}\right)=\neg x_{1} \otimes \neg y_{1}, & \neg\left(x_{1} \oplus y_{1}\right)=\neg x_{1} \oplus \neg y_{1} \\
\neg f & =t \\
\neg t & =f \\
\neg \mathrm{T} & =\mathrm{T} \\
\neg & \perp
\end{aligned}
$$

For logical conflation,

$$
\begin{aligned}
-\left(x_{1} \wedge y_{1}\right)=-x_{1} \wedge-y_{1}, & -\left(x_{1} \vee y_{1}\right)=-x_{1} \vee-y_{1} \\
-\left(x_{1} \otimes y_{1}\right)=-x_{1} \oplus-y_{1}, & -\left(x_{1} \oplus y_{1}\right)=-x_{1} \otimes-y_{1} \\
-f & =f \\
-t & =t \\
-\mathrm{T} & =\perp \\
-\perp & =\mathrm{T}
\end{aligned}
$$

If $B$ is interlaced, then

$$
\begin{gathered}
\perp \wedge \mathrm{T}=f \\
\perp \vee \mathrm{T}=t \\
f \otimes t=\perp \\
f \oplus t=\mathrm{T}
\end{gathered}
$$

The four basic elements of $L_{1} \circ L_{2}$ are the following [20] [25] [34]:

$\perp_{\mathrm{L} \circ \mathrm{L}}=(\inf (L), \inf (L)), \mathrm{T}_{\mathrm{L} \circ \mathrm{L}}=(\sup (L), \sup (L)), t_{\mathrm{L} \circ \mathrm{L}}=(\sup (L), \inf (L))$, and $f_{\mathrm{L} \circ \mathrm{L}}=(\inf (L), \sup (L))$.

\section{Material and Methods}

In this work, our objective is to use concepts of PAL2v to find a logical model 
that is able to simulate phenomena found in quantum mechanics. PAL2v is based on fundamentals that allow some quantum mechanical phenomena to be modeled and so it is a quantum logic, named paraquantum logic (PQL). Therefore, for the construction of a probabilistic paraquantum model ( $p$ PQL-Model) we will associate PQL with the interlaced bilattice FOUR (Belnap's bilattice) and involve probability theory through the Bernoulli distribution.

\section{1. $p$ PQL-Model Associated at Interlaced Bilattice FOUR}

For the construction of a $p$ PQL-Model, we start by associating PQL with the interlaced bilattice FOUR (Belnap's bilattice).

\subsubsection{PQL and Interlaced Bilattice FOUR (Belnap's Bilattice)}

The properties of the interlaced Belnap's bilattice suggest that we can compare outcomes not only from the classical viewpoint, as either being true or false, but also others that may be contradictory.

As was seen in section 4.2, an interlaced bilattice is defined as a bilattice satisfying the condition that the meet and join operations for each partial ordering must be monotonic with respect to the other ordering.

Belnap's four-valued bilattice is an example of a nontrivial interlaced bilattice and it is denoted by $B=\{t, f, \mathrm{~T}, \perp\}$, where $t=1_{\mathrm{t}}$ is true, $f=0_{\mathrm{t}}$ is false, $\mathbf{T}=1_{k}$ is inconsistent (both true and false) or possible, and $\perp=0_{k}$ is paracomplete (neither true nor false) or unknown. These values can be given two natural orders, truth order $\leq_{\mathrm{t}}$ and knowledge order $\leq_{k}$, such that $f \leq_{\mathrm{t}} \mathrm{T} \leq_{\mathrm{t}} t$, $f \leq_{\mathrm{t}} \perp \leq_{\mathrm{t}} t$, and $\perp \leq_{k} f \leq_{k} \mathrm{~T}, \perp \leq_{k} t \leq_{k} \mathrm{~T}$.

Meet and join operators under $\leq_{t}$ are denoted as $\Lambda$ and $V$; they are natural generalizations of the usual conjunction and disjunction notions, so $\mathrm{T} \wedge \perp=f$ and $\mathrm{T} \vee \perp=t$.

Meet and join operators under $\leq_{k}$ are denoted $\otimes$ (consensus, because it produces the most information that two truth values can agree on) and $\oplus$ (gullibility, because it accepts anything it is told), so $f \otimes t=\perp$ and $f \oplus t=\mathrm{T}$.

There is a natural notion of truth negation, denoted as $\neg$ (reverses the $\leq_{t}$ ordering, while preserving the $\leq_{k}$ ordering), switching $f$ and $t$, leaving $\perp$ and $\mathrm{T}$; the corresponding knowledge negation (conflation), denoted as - (reverses the $\leq_{k}$ ordering, while preserving the $\leq_{t}$ ordering), switching $\perp$ and $\mathrm{T}$, leaving $f$ and $t$. In logical analysis, this term "conflation" is used when the identities of two or more distinct objects (individuals, concepts, or logical states) sharing some characteristics of one another appear to be a single identity and the differences appear to become lost.

The fusion of distinct concepts about objects sharing some characteristics tends to obscure analysis of relationships that are emphasized by contrasts.

\subsubsection{Mapping between USCP-Lattice $k$ (USCP: Unit Square in the Cartesian Plane) and Interlaced Bilattice FOUR}

A mapping ( $T$ ) from a complete lattice $L$ to another $L$ is strict iff $T(\perp)=\perp$ 
where $\perp$ is the least element of $L$. As $L$ is a complete lattice, such an element is guaranteed to exist. Mapping $T$ over complete lattices under a specified ordering (such as Belnap's bilattice FOUR) of truth values and where $\neg$ is an injective and strict function from $L$ to $L$ presumes that $L$ contains all elements of $L$.

The mappings between complete bilattices can produce equations that help to find quotients of similarity for analyses of mathematical and efficient data processing [17] [20]. Following these procedures, we will perform a mapping of a complete USCP-lattice $k$ (USCP: unit square in the cartesian plane) to the complete bilattice FOUR (Belnap's bilattice).

As seen in Figure 2, through this mapping, paraconsistent transformations $(P T)$ and logic truth-values represented in bilattice FOUR that can be obtained from USCP-lattice $k$ are produced.

In the mapping USCP-lattice $k \rightarrow$ Belnap's bilattice, three stages are required with actions on the USCP-lattice $k$ to obtain paraconsistent transformations: a) scale expansion, b) rotation by $45^{\circ}$, and c) translation in the $y$ axis [18] [22] [23].

a) Scale expansion: In paraconsistent transformations, the scale is increased in USCP-lattice $k$ as follows: consider point $P(x, y)$ and $P^{\prime}(X, Y)$ as point coordinates after scaling. Scaling entails multiplying each point $P i$ of an object by both a horizontal $\left(S_{x}\right)$ and a vertical $\left(S_{y}\right)$ scale factor. Function $T$ is defined as $T(X p, Y p)=(x p \cdot S x, y p \cdot S y)$.

If $S x=\sqrt{2}$ and $S y=\sqrt{2}$ and $x p=\mu$ and $y p=\lambda$, then the scale increase of USCP-lattice $k$ is given by the first transformation:

$$
T_{1}\left(X_{1}, Y_{1}\right)=(\mu \sqrt{2}, \lambda \sqrt{2})
$$

b) Rotation by $45^{\circ}$ : For paraconsistent transformation, $45^{\circ}$ rotation is made in the USCP-lattice $\kappa$ from its origin; therefore, $T_{2}\left(X_{2}, Y_{2}\right)=\left(X_{1} \cdot \cos \theta-Y_{1} \cdot \sin \theta, X_{1} \cdot \sin \theta+Y_{1} \cos \theta\right)$. For a $45^{\circ}$ rotation,

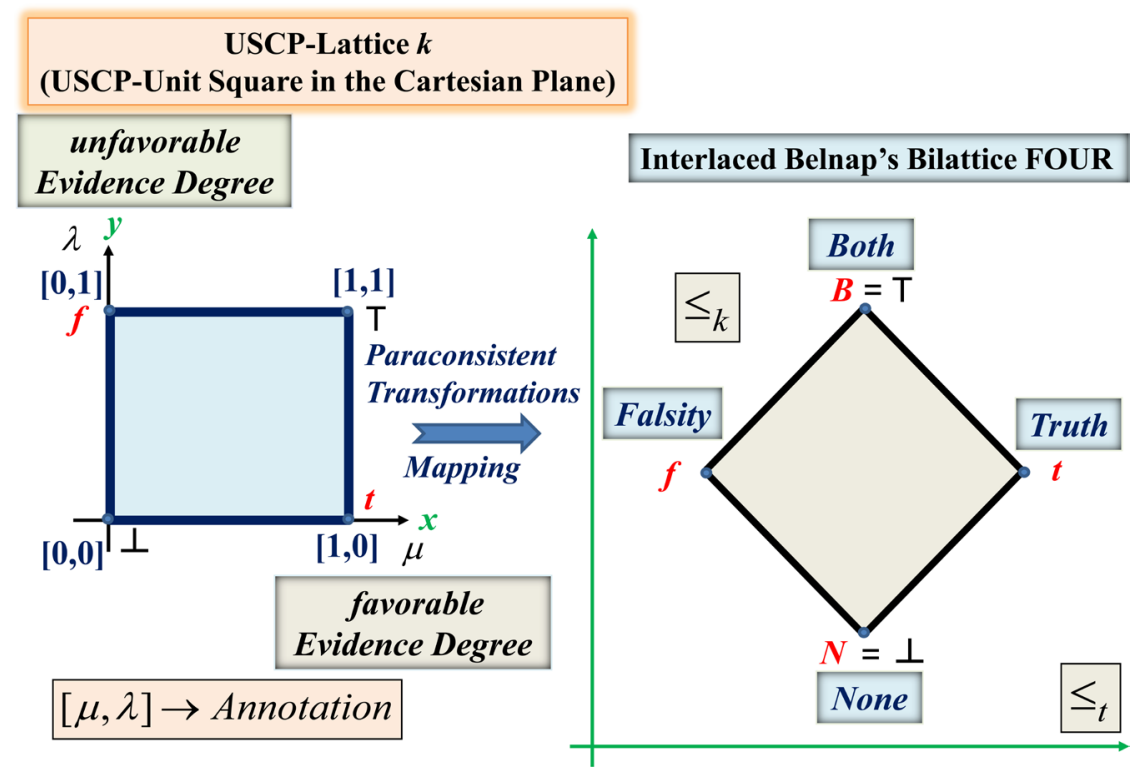

Figure 2. Mapping between a USCP-lattice $k$ to the complete bilattice FOUR. 


$$
T_{2}\left(X_{2}, Y_{2}\right)=\left(X_{1} \cdot \frac{1}{\sqrt{2}}-Y_{1} \cdot \frac{1}{\sqrt{2}}, X_{1} \cdot \frac{1}{\sqrt{2}}+Y_{1} \frac{1}{\sqrt{2}}\right)
$$

From Equation (12), we have $T_{1} ; X_{1}=\mu \sqrt{2}$ and $Y_{1}=\lambda \sqrt{2}$.

$$
\begin{gathered}
X_{2}=\left(\mu \sqrt{2} \frac{1}{\sqrt{2}}-\lambda \sqrt{2} \frac{1}{\sqrt{2}}\right)=(\mu-\lambda) \text { and } \\
Y_{2}=\left(\mu \sqrt{2} \frac{1}{\sqrt{2}}+\lambda \sqrt{2} \frac{1}{\sqrt{2}}\right)=(\mu+\lambda) \\
T_{2}\left(X_{2}, Y_{2}\right)=(\mu-\lambda, \mu+\lambda) ; \text { therefore, } X_{2}=\mu-\lambda \text { and } Y_{2}=\lambda+\mu .
\end{gathered}
$$

c) Translation in the $y$ axis: Consider $T(X p, Y p)=(x p+d x, y p+d y)$, where the total amounts are $d x$ and $d y$. For paraconsistent transformations, a translation in USCP-lattice $\kappa$ is made on its $y$ axis as follows: let $T_{2}\left(X_{2}, Y_{2}\right)=(\mu-\lambda, \mu+\lambda)$; thus, $x p=\mu-\lambda$ and $y p=\mu+\lambda$. With $d x=0$ and $d y=-1$, the final transformation is

$$
T_{3}\left(X_{3}, Y_{3}\right)=(\mu-\lambda, \mu+\lambda-1)
$$

Therefore, $X_{3}=\mu-\lambda$ and $Y_{3}=\mu+\lambda-1$.

\subsection{Equations of Certainty and Contradiction Degree in the Interlaced Bilattice FOUR}

The representation of degrees of evidence in the interlaced bilattice FOUR (Belnap's bilattice) is made considering the certainty degree $(D c)$ from Equation (14) as $\mathrm{X} 3$ and the contradiction degree $(D c t)$ as $\mathrm{Y} 3$ [18] [22] [23].

$$
\begin{aligned}
& X_{3}=D c \rightarrow \text { Certainty degree } \\
& \qquad D c=\mu-\lambda \\
& Y_{3}=D c t \rightarrow \text { Contradiction degree } \\
& D c t=\mu+\lambda-1
\end{aligned}
$$

With this mapping on the interlaced bilattice FOUR, the equations are inserted in a set of complex numbers $C$, where the values of the certainty degree $D c$ will be exposed on the $x$-horizontal axis (real) and the values of the contradiction degree Dct exposed on the $y$-vertical axis (imaginary). Figure 3 shows the mapping of the USCP-lattice $k$, where the degrees of favorable $(\mu)$ and unfavorable $(\lambda)$ evidence are exposed, to the complete bilattice FOUR (Belnap's bilattice), where the certainty degree $D c$ (horizontal real axis) and contradiction degree $D c t$ (vertical imaginary axis) are exposed [18] [22] [23].

A paraconsistent logical state $\psi$ is considered as the point of intersection between the certainty degree $D c$ and the contradiction degree $D c t$ located in the interlaced bilattice FOUR. Therefore, the representation of a paraconsistent logical state $\psi$, will be [22] [23]:

$$
\psi=(D c, D c t)
$$

\subsection{Action of Logical Negation in the Interlaced Bilattice FOUR}

We consider that $[\mu, \lambda]$ is the annotation that belongs to the set $B$ of interlaced 


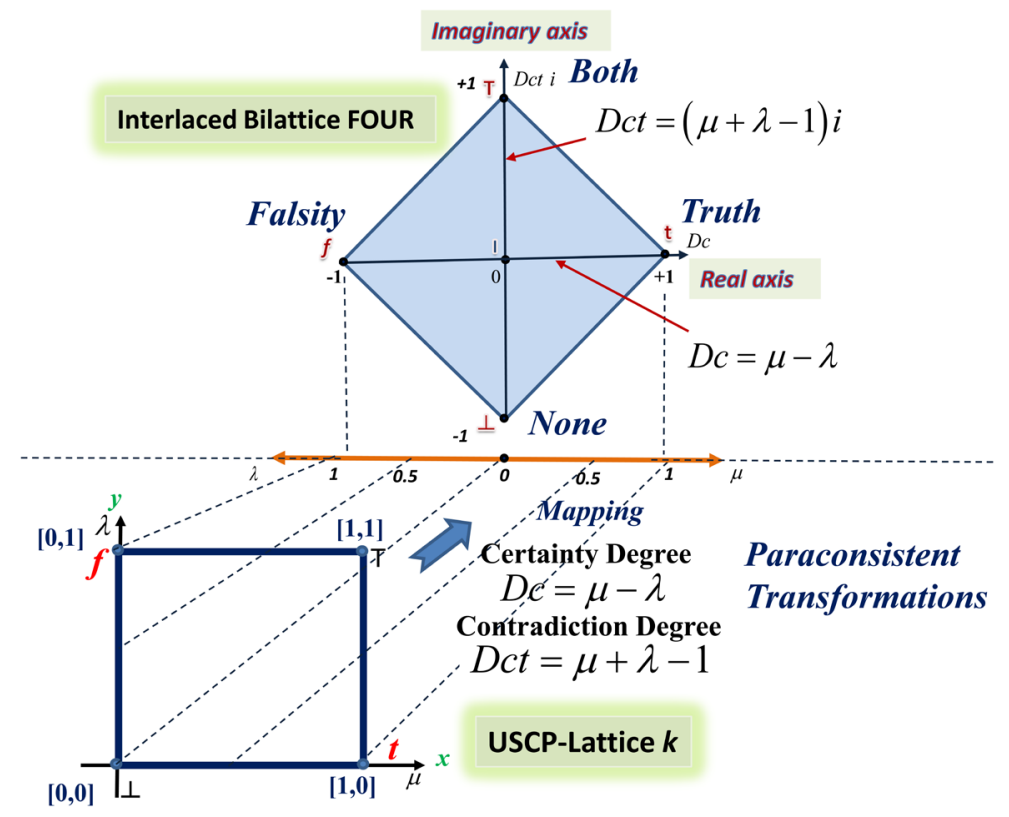

Figure 3. Mapping between a USCP-lattice $k$ to the complete bilattice FOUR.

bilattice FOUR, such that $[\mu, \lambda] \in \operatorname{set} B=\{t, f, \mathrm{~T}, \perp\}$.

As the evidence degrees $\mu, \lambda \in[1,0] \subset \Re$, then for each operation of logical negation (Equation (7)) the certainty degree (Equation (15)) changes the sign and the contradiction degree (Equation (16)) remains. Therefore, in the action of logical negation operation $\neg[\mu, \lambda]=[\lambda, \mu]$, we have the following:

For $+D c t$,

if $+D c$, then it changes to $-D c$ and, from Equation (17), the logical state that was $\psi=(+D c,+D c t)$ changes to $\neg \psi=(-D c,+D c t)$;

if $-D c$, then it changes to $+D c$ and, from Equation (17), the logical state that was $\psi=(-D c,+D c t)$ changes to $\neg \psi=(+D c,+D c t)$.

For $-D c t$,

if $+D c$, then it changes to $-D c$ and, from Equation (17), the logical state that was $\psi=(+D c,-D c t)$ changes to $\neg \psi=(-D c,-D c t)$;

if $-D c$, then it changes to $+D c$ and, from Equation (17), the logical state that was $\psi=(-D c,-D c t)$ changes to $\neg \psi=(+D c,-D c t)$.

\subsection{Action of Logical Conflation in the Interlaced Bilattice FOUR}

The logical conflation is a logical operation that the interlaced Belnap's bilattice admits.

We consider that $(x ; y)$ is the truth values, where $(x ; y)$ belong to the set $B$ of interlaced bilattice FOUR.

The conflation operation - is represented by $-(x, y)=(1-y, 1-x)$, and for PQL and the interlaced Belnap's Bilattice FOUR associated with it, we have

$$
-[\mu, \lambda]=[1-\lambda, 1-\mu]
$$

where $[\mu, \lambda]$ is the annotation (truth values), such that 
$[\mu, \lambda] \in \operatorname{set} B=\{t, f, \mathrm{~T}, \perp\} \quad$ of interlaced bilattice FOUR.

As the evidence degrees $\mu, \lambda \in[1,0] \subset \Re$, for each logical conflation operation of Equation (18) the contradiction degree (Equation (16)) changes the sign and the certainty degree (Equation (15)) remains. Therefore, for the action of logical conflation operation $-[\mu, \lambda]=[1-\mu, 1-\lambda]$, we have the following:

For $+D c$,

if $+D c t$, then it changes to $-D c t$ and, from Equation (17), the logical state that was $\psi=(+D c,+D c t)$ changes to $-\psi=(+D c,-D c t)$;

if $-D c t$, then it changes to $+D c t$ and, from Equation (17), the logical state that was $\psi=(+D c,-D c t)$ changes to $-\psi=(+D c,+D c t)$.

For $-D c$,

if $+D c t$, then it changes to $-D c t$ and, from Equation (17), the logical state that was $\psi=(+D c,-D c t)$ changes to $\psi=(-D c,-D c t)$;

if $-D c t$, then it changes to $+D c t$ and, from Equation (17), the logical state that was $\psi=(-D c,-D c t)$ changes to $\psi=(-D c,+D c t)$.

\subsection{Representation of the Probabilistic Paraquantum Logical Model (pPQL-Model)}

The representation of the $p$ PQL-Model is built from modeling the USCP-lattice $k$ with the probability values of the Bernoulli distribution. In this way, the USCP-lattice $k$ mapping will apply the probabilistic values on the interlaced bilattice FOUR (Belnap's bilattice). In the $p$ PQL-Model, the probability $p$ is an outcome that generates the degrees of evidence for the analysis of a proposition $P$ for affirmation (true) or refutation (false). For example, we can relate the proposition $P$ with a probability mass function (pmf) of the Bernoulli distribution (Equation (3)) with the $k=1$ success (result $\mathrm{H}$-heads) and $k=0$ failure (result T-tails).

The results in linear variation and relationship determined by the variance $\sigma^{2}$ with Equation (5) can be seen in Figure 4.

As in PQL theory, an annotation is composed of the two values of degrees of evidence at probability $p$, then for application of degrees of evidence in the $p P Q L-M o d e l$, we have the following:

With $k=0$, the pmf generates the values for the unfavorable evidence degree at probability $p$.

With $k=1$ the pmf generates the values for the favorable evidence degree at probability $p$.

The degrees of evidence ( $\mu$ and $\lambda$ ) that will form the annotation are extracted from the pmf obeying the Bernoulli distribution for a single Bernoulli trial (launch of a coin) or for two Bernoulli trials (launch of two coins) simultaneously.

\subsubsection{Representation at Bilattice FOUR of a Single Bernoulli Trial}

For a single Bernoulli trial (launch of one coin), the representation can be made considering that the variance $\sigma^{2}$ has values obtained by Equation (5), where a 


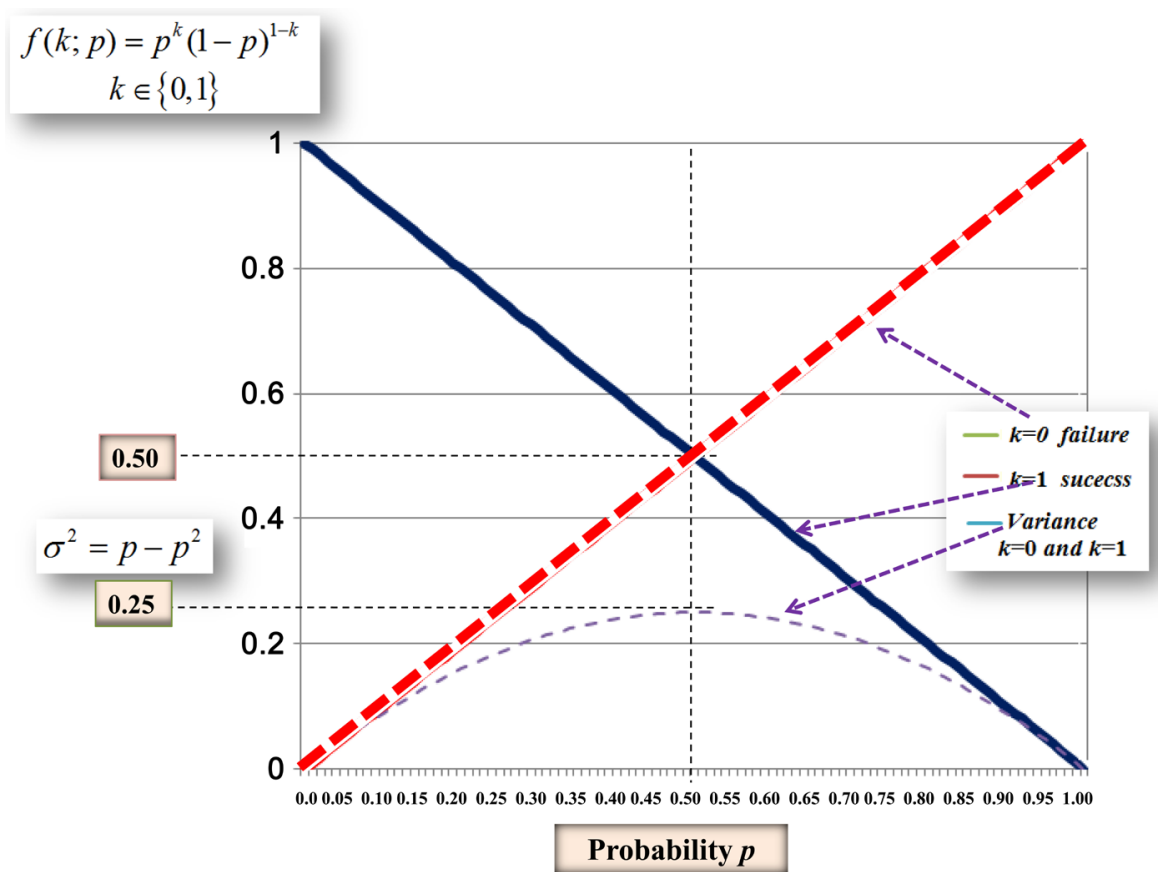

Figure 4. Results of the application of the probability mass function (pmf) of the Bernoulli distribution and variance for $k=1$ and $k=0$.

maximum value 0.25 for a function of $p \in[0,1]$ resulted in a parabola curve (as shown in Figure 4). For the initial modeling, we can consider that a greater variance value means that a conclusion on the logical state that results in True $(t)$ or in False $(f)$ will be made with a higher degree of uncertainty. This situation is represented by the PQL through the certainty degree $(D c)$ equal to zero (Equation (15)). Otherwise, a minor variance value leads to a conclusion on the logical state that results in True $(t)$ with the certainty degree $(D c)$ equal to 1 or results in False $(f)$ with the certainty degree $(D c)$ equal to -1 .

It can be assumed that the increase in the variance value reduces the value of the certainty degree $\left(D_{c}\right)$ in both logical states, True $(t)$ and False $(f)$. In this way, the value of the variance, when related to the $p$ PQL-Model, will be compared to the values of the degrees of unfavorable evidence at probability $p$. As changes in probability $p$ are represented by two straight lines generated by the pmf (Equation (3)), the relationship with the favorable degree of evidence at probability $p$ is represented by the complementary value.

In the Bernoulli distribution, the standard deviation $\sigma$ (Equation (6)) and the variance $\sigma^{2}$ (Equation (5)) have the same characteristics related at evidence either favorable or unfavorable to probability $p$. Then, for a better representation in the bilattice FOUR, we use the standard deviation $\sigma$ in the equations.

Under these conditions, the degrees of evidence will be represented in the $p P Q L-M o d e l$ by the following equations.

The degree of unfavorable evidence at probability $p$ is to the True logical state $(t): \lambda_{(p) t}=\sqrt{\left(p-p^{2}\right)}$.

The degree of favorable evidence at probability $p$ is the complement: 
$\mu_{(p) t}=1-\sqrt{\left(p-p^{2}\right)}$.

The maximum value for the standard deviation $\sigma$ will be for probability $p=$ 0.5 . Then, it will be $\sqrt{\sigma^{2}}=0.5$, which leads to the degrees of evidence at a range variation from 0 to 0.5 . Therefore, a constant value of 0.5 will be added so that the degrees of evidence can reach a complete unitary value. With these assumptions, the extraction mode of evidence degrees results in the following:

In relation to the True logical state $(t)$, the favorable degree of evidence at probability $p$ is

$$
\mu_{(p) t}=\frac{1}{2}+\sqrt{\left(p-p^{2}\right)}
$$

and the unfavorable degree of evidence at probability $p$ is

$$
\lambda_{(p) t}=\frac{1}{2}-\sqrt{\left(p-p^{2}\right)}
$$

In relation to the False logical state $(f)$, the favorable degree of evidence at probability $p$ is

$$
\mu_{(p) f}=\frac{1}{2}-\sqrt{\left(p-p^{2}\right)}
$$

and the unfavorable degree of evidence at probability $p$ is

$$
\lambda_{(p) f}=\frac{1}{2}+\sqrt{\left(p-p^{2}\right)}
$$

Then, for the separate analysis, we have the following:

a) For the True logical state $(t)$, the degree of favorable evidence $(\mu)$ at probability $p$ (Equation (19)) and the degree of unfavorable evidence $(\lambda)$ at probability $p$ (Equation (20)):

From Equation (15), the certainty degree is a function of $p$ and it is represented by

$$
D c_{(p) t}=\mu_{(p) t}-\lambda_{(p) t}, \text { where } 0 \leq D c_{(p) t} \leq+1 .
$$

From Equation (16), as $\mu_{(p) t}+\lambda_{(p) t}=1$ for any value of $p$, the contradiction degree will always be null. The paraquantum logical state is a function of $p$ and it is represented from Equation (17) by

$$
\psi_{(p) t}=\left(D c_{(p) t}, D c t_{(p) t}\right) \rightarrow \psi_{(p) t}=\left(+D c_{(p) t}, 0\right), \text { where } 0 \leq D c_{(p) t} \leq+1 .
$$

Figure 5 shows the extraction mode of degrees of evidence through the pmf of the Bernoulli distribution.

For example, the computed values of evidence degrees at probability $p=0.5$ are $\mu_{(p) t}=\frac{1}{2}+\sqrt{p-p^{2}}=\frac{1}{2}+\sqrt{0.5-0.5^{2}}=1$ and $\lambda_{(p) t}=\frac{1}{2}-\sqrt{p-p^{2}}=\frac{1}{2}-\sqrt{0.5-0.5^{2}}=0$

The annotation $[1,0] \rightarrow D c_{(p) t}=1$ and $D c t_{(p) t}=0 \rightarrow \psi_{(p) t}=(+1,0)$.

The computed values of evidence degrees at probability $p=1$ and $p=0$ are $\mu_{(p) t}=\frac{1}{2}$ and $\lambda_{(p) t}=\frac{1}{2}$. The annotation $[0.5,0.5] \rightarrow D c_{(p) t}=0$ and 


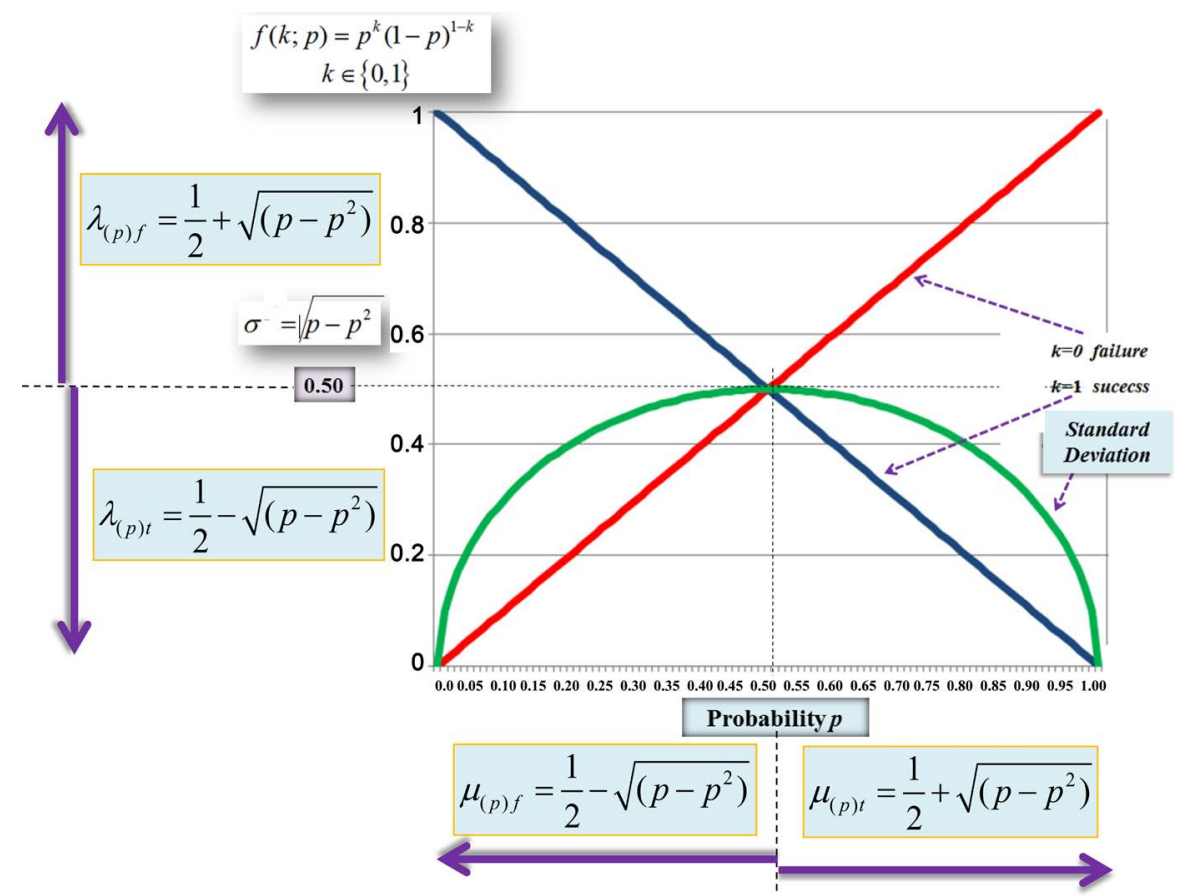

Figure 5. Extraction mode of degrees of evidence through the probability mass function (pmf) of the Bernoulli distribution.

$\operatorname{Dct}_{(p) t}=0 \rightarrow \psi_{(p) t}=(0,0)$.

b) For the False logical state $(f)$ the degree of favorable evidence $(\mu)$ at probability $p$ (Equation (21)) and the degree of unfavorable evidence $(\lambda)$ at probability $p$ (Equation (22)):

The certainty degree is a function of $p$ and it is represented from Equation (15) by

$$
D c_{(p) f}=\mu_{(p) f}-\lambda_{(p) f}, \text { where }-1 \leq D c_{(p) f} \leq 0 .
$$

From equation (16), as $\mu_{(p) f}+\lambda_{(p) f}=1$, the contradiction degree will always be null. The paraquantum logical state is a function of $p$ and it is represented from equation (17) by

$$
\psi_{(p) f}=\left(D c_{(p) f}, D c t_{(p) f}\right) \rightarrow \psi_{(p) f}=\left(D c_{(p) f}, 0\right), \text { where }-1 \leq D c_{(p) f} \leq 0 .
$$

For example, the computed values of evidence degrees at probability $p=0.5$ are

$$
\begin{gathered}
\mu_{(p) f}=\frac{1}{2}-\sqrt{p-p^{2}}=\frac{1}{2}-\sqrt{0.5-0.5^{2}}=0 \text { and } \\
\lambda_{(p) f}=\frac{1}{2}+\sqrt{p-p^{2}}=\frac{1}{2}+\sqrt{0.5-0.5^{2}}=1
\end{gathered}
$$

The annotation $[1,0] \rightarrow D c_{(p) f}=-1$ and $D c t_{(p) f}=0 \quad \psi_{(p) f}=(0,-1)$.

The computed values of evidence degrees at probability $p=1$ and $p=0$ are $\mu_{(p) f}=\frac{1}{2}$ and $\lambda_{(p) f}=\frac{1}{2}$. The annotation $[0.5,0.5] \rightarrow D c_{(p) t}=0$ and $\operatorname{Dct}_{(p) t}=0 \rightarrow \psi_{(p) t}=(0,0)$. 
For a single Bernoulli trial (launch of one coin), there are two paraquantum logical states in the function of $p$. The probabilistic paraquantum state of the True logical State $(t)$ is represented only by $D c_{(p) t}$ and the probabilistic paraquantum state of the False logical state $(f)$ is represented only by $D c_{(p) f}$. For the maximum and minimum variations of the pmf of the Bernoulli distribution, the contradiction degree $(D c t)$ will always be zero with $0 \leq D c_{(p) t} \leq+1$ and $-1 \leq D c_{(p) f} \leq 0$.

The representation for the probability values ranging from 0 to 1 are generated by the pmf of the Bernoulli distribution. For a better analysis, we consider that the variation of the probability begins with an initial value $p=0.5$; then for each probability value $(p)$ that decreases, the corresponding complementary value $(q=1-p)$ will increase.

The variation of the probability values occurs along with changes in the values of the variance $\sigma^{2}$ and standard deviation $\sigma$. With the reduction in variance, both degrees of certainty $\left(D_{C(p) f}\right.$ and $\left.D_{C(p) t}\right)$ increase simultaneously toward the True logical state $(t)$ and the False logical state $(f)$. Similarly, with the increase in variance, both degrees of certainty $\left(D_{C(p) f}\right.$ and $\left.D_{C(p) t}\right)$ approach the undefined logical state (I) located at the point equidistant from the vertices of the bilattice FOUR. At the point of the undefined logical state (I) is the maximum variance with both degrees of favorable and unfavorable evidence equal to 0.5 , which results in both degrees of certainty with zero values $\left(\left(D_{C(p) f}=D_{C(p) t}=0\right)\right.$.

Figure 6 shows the representation of the single Bernoulli trial on paraquantum analysis using interlaced bilattice FOUR.

\subsection{Indistinguishability between Paraquantum Logical States}

In the Paraquantum analysis, before the logical state is set between True $(t)$ and

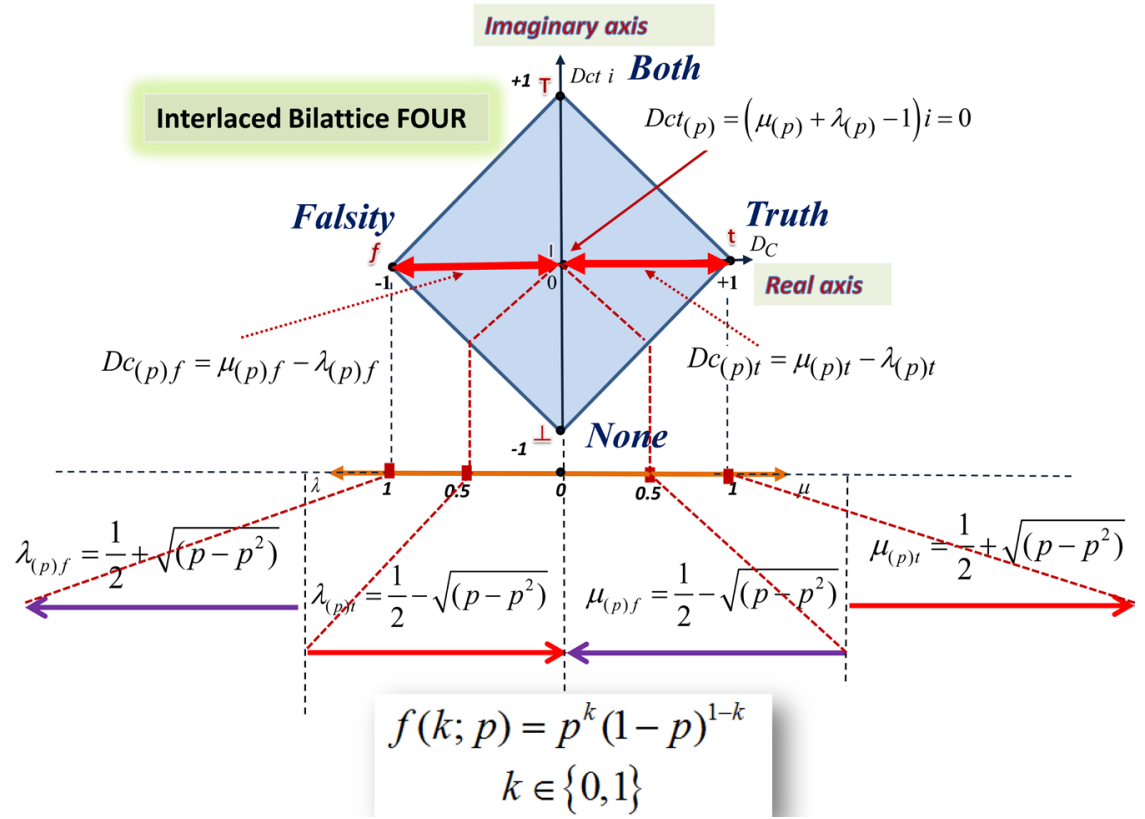

Figure 6. Representation of the single Bernoulli trial using interlaced bilattice FOUR. 
False $(f)$, the variance $\sigma^{2}$ causes indistinguishability between the two.

This indistinguishability between the True logical state $(t)$ and the False logical state $(f)$ is represented by the exchange of position between the degrees of favorable $(\mu)$ and unfavorable $(\lambda)$ evidence; this effect is due to the logical negation represented in Equation (7) and the logical conflation represented in Equation (18). In this condition, the two logical states, True $(t)$ and False $(f)$, are in superposition and in a state of entanglement.

\subsubsection{Action of Logical Negation for the Single Bernoulli Trial on the Interlaced Bilattice FOUR}

The actions of operation of logical negation are described as follows:

For the True logical state $(t)$, the operation of logical negation from Equation (7) with evidence degrees of Equations ((19), (20)) are:

$$
\begin{aligned}
& \neg\left[\mu_{(p) t}, \lambda_{(p) t}\right]=\left[\lambda_{(p) t}, \mu_{(p) t}\right] \\
& \neg\left[\frac{1}{2}+\sqrt{\left(p-p^{2}\right)}, \frac{1}{2}-\sqrt{\left(p-p^{2}\right)}\right]=\left[\frac{1}{2}-\sqrt{\left(p-p^{2}\right)}, \frac{1}{2}+\sqrt{\left(p-p^{2}\right)}\right] \quad(23 \mathrm{a}) \\
& \neg\left[\left(0.5 \leq \mu_{(p) t} \leq 1.0\right),\left(0.0 \leq \lambda_{(p) t} \leq 0.5\right)\right]=\left[\left(0.0 \leq \lambda_{(p) t} \leq 0.5\right),\left(0.5 \leq \mu_{(p) t} \leq 1.0\right)\right]
\end{aligned}
$$

For the logical False state $(f)$, the operation of logical negation from Equation (7) with evidence degrees of Equations ((22), (23)) are:

$$
\begin{aligned}
& \neg\left[\mu_{(p) f}, \lambda_{(p) f}\right]=\left[\lambda_{(p) f}, \mu_{(p) f}\right] \\
& \neg\left[\frac{1}{2}-\sqrt{\left(p-p^{2}\right)}, \frac{1}{2}+\sqrt{\left(p-p^{2}\right)}\right]=\left[\frac{1}{2}+\sqrt{\left(p-p^{2}\right)}, \frac{1}{2}-\sqrt{\left(p-p^{2}\right)}\right] \quad(23 \mathrm{~b}) \\
& \neg\left[\left(0.0 \leq \mu_{(p) t} \leq 0.5\right),\left(0.5 \leq \lambda_{(p) t} \leq 1.0\right)\right]=\left[\left(0.5 \leq \lambda_{(p) t} \leq 1.0\right),\left(0.0 \leq \mu_{(p) t} \leq 0.5\right)\right]
\end{aligned}
$$

Therefore, variations in degrees of evidence happen in a complementary manner with the logical negation operation.

\subsubsection{Action of Logical Conflation for the Single Bernoulli Trial on the Interlaced Bilattice FOUR}

The actions of the operation of logical conflation are described as follows:

For the True logical state $(t)$, the operation of logical conflation from Equation (18) with evidence degrees of Equations ((19), (20)) are:

$$
\begin{gathered}
-\left[\mu_{(p) t}, \lambda_{(p) t}\right]=\left[1-\mu_{(p) t}, 1-\lambda_{(p) t}\right] \\
-\left[\frac{1}{2}+\sqrt{\left(p-p^{2}\right)}, \frac{1}{2}-\sqrt{\left(p-p^{2}\right)}\right]=\left[1-\left(\frac{1}{2}-\sqrt{\left(p-p^{2}\right)}\right), 1-\left(\frac{1}{2}+\sqrt{\left(p-p^{2}\right)}\right)\right] \quad(24 \mathrm{a}) \\
-\left[\left(0.5 \leq \mu_{(p) t} \leq 1.0\right),\left(0.0 \leq \lambda_{(p) t} \leq 0.5\right)\right]=\left[\left(0.5 \leq \mu_{(p) t} \leq 1.0\right),\left(0.0 \leq \lambda_{(p) t} \leq 0.5\right)\right]
\end{gathered}
$$

For the False logical state $(f)$, the operation of logical conflation from Equation (18) with evidence degrees of Equations ((21), (22)) are:

$$
\begin{gathered}
-\left[\mu_{(p) f}, \lambda_{(p) f}\right]=\left[1-\mu_{(p) f}, 1-\lambda_{(p) f}\right] \\
-\left[\frac{1}{2}-\sqrt{\left(p-p^{2}\right)}, \frac{1}{2}+\sqrt{\left(p-p^{2}\right)}\right]=\left[1-\left(\frac{1}{2}+\sqrt{\left(p-p^{2}\right)}\right), 1-\left(\frac{1}{2}-\sqrt{\left(p-p^{2}\right)}\right)\right]
\end{gathered}
$$




$$
-\left[\left(0.0 \leq \mu_{(p) t} \leq 0.5\right),\left(0.5 \leq \lambda_{(p) t} \leq 1.0\right)\right]=\left[\left(0.0 \leq \mu_{(p) t} \leq 0.5\right),\left(0.5 \leq \lambda_{(p) t} \leq 1.0\right)\right]
$$

Therefore, in the single Bernoulli trial, there is no difference in the variation of the degrees of evidence with the operation of the logical conflation. This means that the operation of the conflation has no logical action because there is no contradiction in this situation.

For a single Bernoulli trial that does not have any inconsistencies, the superposition state can be calculated using the degree of contradiction $\left(D_{c t}\right)$ (Equation (16)), and the value remains null. The uncertainty could also be estimated by the degree of certainty $\left(D_{C}\right)$ (Equation (15)); however, the double values of degrees of evidence that continuously change position induce indistinguishability and produce the uncertainty value corresponding to the zero degree of certainty.

\subsection{Representation of Two Bernoulli Trials in Simultaneous Mode at Interlaced Bilattice FOUR}

In the representation, in an interlaced bilattice FOUR (Belnap's bilattice) for two Bernoulli trials in simultaneous mode (launch of two coins), two variances are considered based on the following theorem [32]:

If $X$ and $Y$ are independent random variables, then

$$
\operatorname{Var}(X+Y)=\operatorname{Var}(X)+\operatorname{Var}(Y)
$$

This proof relies on $E(X+Y)=E(X) E(Y)$ when $X$ and $Y$ are independent.

Therefore, with two Bernoulli trials and Equation (35), one can consider the double variance as $\sigma_{X, Y}^{2}=\left(p_{X}-p_{X}^{2}\right)+\left(p_{Y}-p_{Y}^{2}\right)$, where the double standard deviation will be

$$
\sigma_{X, Y}=\sqrt{\sigma_{X, Y}^{2}} \text { or } \sigma_{X, Y}=\sqrt{\left(p_{X}-p_{X}^{2}\right)+\left(p_{Y}-p_{Y}^{2}\right)} .
$$

Considering the probability values $p_{X}=p_{Y}=p$,

$$
\sigma_{X, Y}=\sqrt{2 \sigma^{2}}
$$

Equation (26) can be rewritten as $\sigma_{X, Y}=\sqrt{2} \sqrt{\sigma^{2}}$,or, from Equation (6), considering the single standard deviation $\sigma$

$$
\sigma_{X, Y}=\sqrt{2} \sigma
$$

Matching (26) and (27), we have

$$
\sqrt{2 \sigma^{2}}=\sqrt{2} \sigma
$$

Multiplying by 2 on both sides of the equation, we can make an adjustment to the value of the probability $p$ (the area under the parabolic curve in Figure 6) obtained by the pmf of the Bernoulli distribution: $2 \sqrt{2 \sigma^{2}}=2 \sqrt{2} \sigma$. The last expression also can be rewritten (taking into consideration the single standard deviation $\sigma$ from Equation (27)) as $\frac{2}{\sqrt{2}}\left(\sqrt{2 \sigma^{2}}\right)=2 \sigma$ or 


$$
\frac{1}{\sqrt{2}}\left(\sqrt{2\left(p-p^{2}\right)}\right)=\sqrt{\left(p-p^{2}\right)}
$$

Equation (29) relates the values of the function of the probability $p$ and represents the area under the parabolic curve in Figure 6. Therefore, for defines $\mu_{(p)}$, we can make the following analysis:

- when $p=0.5$ the Variance value is $\sigma^{2}=0.25 \rightarrow \sigma=0.5$. Result $\mu_{(p)}=2 \sigma=1$

- when $p=1.0$ the Variance value is $\sigma^{2}=0.0 \rightarrow \sigma=0.0$ Result $\mu_{(p)}=2 \sigma=0$

- when $p=0.0$ the Variance value is $\sigma^{2}=0.0 \rightarrow \sigma=0.0$ Result $\mu_{(p)}=2 \sigma=0$

For $\sigma^{2}=0.0625 \rightarrow p \cong 0.067$ and $1-p \cong 0.933$

$\sigma=0.25 \rightarrow 2 \sigma=0.5 \rightarrow \sqrt{\sigma}=0.5$. We define $\mu_{(p)}=2 \sigma$, and for representation of $\lambda_{(p)}$, we chosen a value that is 0.5 in this same condition. Therefore, the equations that adapt in this condition are $\mu_{(b)}=2 \sqrt{p-p^{2}}=2 \sigma$ and $\lambda_{(D)}=\sqrt{\sqrt{p-p^{2}}}=\sqrt{\sigma}$.

Figure 7 shows the graphs of $\lambda_{(p)}$ and $\mu_{(p)}$ as a function of $\mathrm{p} \in[0,1]$ that results in its two different curves.

The largest value occurs when $p=0.5$ and the smallest value is 0 when $p=0$ and/or $p=1$. There are two encounter points in probability $p=0.067$ and $p=$ 0.933 that mark the start and final for valid analyses in PQL.

We can consider Equation (29) as a generator of favorable and unfavorable degrees of evidence at probability $p$. In this condition, the two degrees $\mu$ and $\lambda$ are generated in simultaneous mode for the two logical states, True $(t)$ and False $(f)$. This means that for two Bernoulli trials for the True logical state $(t)$, two degrees of unfavorable evidence at probability $p\left(\lambda_{p t}\right)$ and two degrees of favorable evidence at probability $p\left(\mu_{p t}\right)$ are generated.

$$
\begin{aligned}
& \mu_{(p) t 1}=2 \sigma \\
& \lambda_{(p) t 2}=\sqrt{\sigma}
\end{aligned}
$$

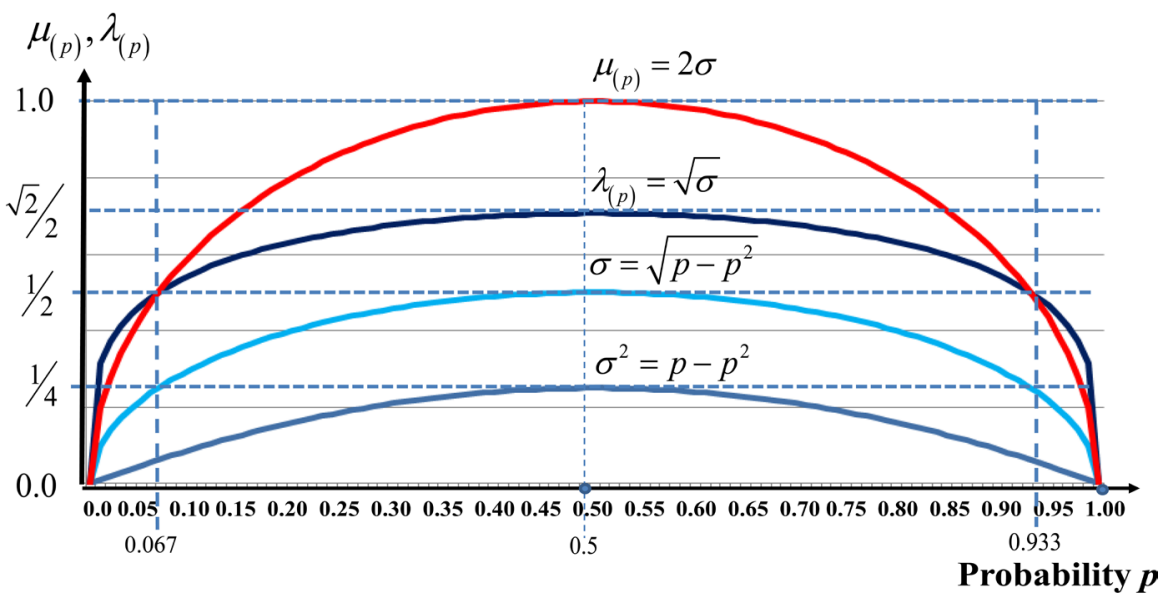

Figure 7. The graphs of $\lambda_{(p)}$ and $\mu_{(p)}$ as a function of $p \in[0,1]$. 


$$
\begin{aligned}
& \mu_{(p) t 2}=1-\sqrt{\sigma} \\
& \lambda_{(p) t 1}=1-2 \sigma
\end{aligned}
$$

For the False logical state $(f)$, two degrees of favorable evidence at probability $p\left(\mu_{p t}\right)$ and two degrees of unfavorable evidence at probability $p\left(\lambda_{p t}\right)$ are generated.

$$
\begin{aligned}
& \lambda_{(p) f 1}=2 \sigma \\
& \mu_{(p) f 2}=\sqrt{\sigma} \\
& \mu_{(p) f 1}=1-2 \sigma \\
& \lambda_{(p) f 2}=1-\sqrt{\sigma}
\end{aligned}
$$

In the mapping, these values originating at probability $p$ are represented on the horizontal $(x)$ and vertical $(y)$ axes of the USCP-lattice $k$ in the formatting of the degrees of favorable evidence $(\mu)$ and unfavorable evidence $(\lambda)$, respectively. With the two corresponding degrees of evidence (favorable and unfavorable at probability $p$ ), the paraquantum logical states $\psi$ in the interlaced bilattice FOUR on superposition states are obtained. This mapping is made through paraconsistent transformations and results in the interlaced bilattice FOUR with representations of superposed paraquantum logical states $\left(\psi_{\text {sup }}\right)$ in the interior.

The application of mapping results in four paraquantum logical states $\psi_{\text {sup }}$ formed with values of degrees of certainty $(D c)$ from Equation (15) and degrees of contradiction $(D c t)$ from Equation (16). The set of states in this configuration that undergo the changes in $D c_{(p)}$ and $D c t_{(p)}$ values vary their internal positions in the interlaced bilattice FOUR. This set of superposed logical states $\psi_{\text {sup }}$ is obtained as follows:

With Equations ((30) and (31)), from Equation (15), we have the probabilistic certainty degree

$$
D c_{(p) t 1}=\mu_{(p) t 1}-\lambda_{(p) t 2}
$$

and from Equation (16), we have the probabilistic contradiction degree

$$
\operatorname{Dct}_{(p) t 1}=\mu_{(p) t 1}+\lambda_{(p) t 2}-1
$$

These two values form a pair at a single point in the bilattice FOUR - a single superposed paraquantum logical state-as represented in Equation (17):

$$
\psi_{(P Q) 1}=\left(D c_{(p) t 1}, D c t_{(p) t 1}\right)
$$

With Equations ((32) and (33)), from Equation (15), we have the probabilistic certainty degree $D c_{(p) t 2}=\mu_{(p) t 2}-\lambda_{(p) t 1}$ and from Equation (16), we have the probabilistic contradiction degree

$$
\operatorname{Dct}_{(p) t 2}=\mu_{(p) t 2}+\lambda_{(p) t 1}-1
$$

These two values form a pair at a single point in the bilattice FOUR - a single superposed Paraquantum logical state-as represented in Equation (17): 


$$
\psi_{(P Q) 2}=\left(D c_{(p) t 2}, D c t_{(p) t 2}\right)
$$

With Equations ((34) and (35)), from Equation (15), we have the probabilistic certainty degree

$$
D c_{(p) f 1}=\mu_{(p) f 1}-\lambda_{(p) f 2}
$$

and from Equation (16), we have the probabilistic contradiction degree

$$
\operatorname{Dct}_{(p) f 1}=\mu_{(p) f 1}+\lambda_{(p) f 2}-1
$$

These two values form a pair at a single point in the bilattice FOUR-a single superposed paraquantum logical state-as represented in Equation (17):

$$
\psi_{(P Q) 3}=\left(D c_{(p) f 1}, D c t_{(p) f 1}\right)
$$

With Equations ((36) and (37)), from Equation (15), we have the probabilistic certainty degree

$$
D c_{(p) f 2}=\mu_{(p) f 2}-\lambda_{(p) f 1}
$$

and from Equation (16), we have the probabilistic contradiction degree

$$
\operatorname{Dct}_{(p) f 2}=\mu_{(p) f 2}+\lambda_{(p) f 1}-1
$$

These two values form a pair at a single point in the bilattice FOUR - a single superposed Paraquantum logical state-as represented in Equation (17):

$$
\psi_{(P Q) 4}=\left(D c_{(p) f 2}, D c t_{(p) f 2}\right)
$$

The set of superposed paraquantum logical states is

$$
\psi_{\sup (p)}=\left\{\psi_{\sup (P Q) 1}, \psi_{\sup (P Q) 2}, \psi_{\sup (P Q) 3}, \psi_{\sup (P Q) 4}\right\}
$$

A vector of state $\mathrm{P}(\psi)$ in the interlaced bilattice FOUR originates in one of the two vertices, True $(t)$ or False $(f)$, which compose the certainty degree horizontal axis. With its origin in one of the vertices of the bilattice FOUR, the vector of state $\mathrm{P}(\psi)$ has at its end a point formed by the pair $\left(\psi_{(\mathrm{PO})}=\left(D_{C}, D_{c t}\right)\right.$ indicated by the paraquantum logical state (Equation (17)). Paraquantum logical states $\psi$ in the trajectory indicated by the vertex of state vector $\mathrm{P}(\psi)$ of unitary module are defined as superposed paraquantum logical states $\psi_{\text {sup }}$. In the interlaced bilattice FOUR, each superposed paraquantum logical state has variations established by four state vectors of unitary module. For this one-dimensional space study, we divided the bilattice FOUR into four quadrants. For example, in the quadrant I of bilattice FOUR, the state vector $\mathrm{P}(\psi)$ will always be the sum of its two component vectors:

Vector $\boldsymbol{X}_{C}$, with the same direction of the certainty degree axis (horizontal), whose module equals the intensity complement of the certainty degree: $X_{C}=1-\left|D c_{(p) t 1}\right|$

Vector $\boldsymbol{Y}_{c t}$, with the same direction of the contradiction degree axis (vertical), whose module equals the intensity of the contradiction degree: $Y_{c t}=D c t_{(p) t 2}$

Therefore, given any single superposed paraquantum logical state $\left(\psi_{\text {cur }}\right)$ as de- 
fined in the set presented in Equation (49), we can calculate the module of vector of state $\mathrm{P}(\psi)$ according to the equation:

$$
\operatorname{MP}(\psi)=\sqrt{\left(1-\left|D c_{(p)}\right|\right)^{2}+D c t_{(p)}^{2}}
$$

where $D c_{(p)}=$ probabilistic certainty degree calculated by (15),

$D c t_{(p)}=$ probabilistic contradiction degree calculated by (16) and $\left(1-\left|D c_{(p)}\right|\right)^{2}+D c t_{(p)}^{2}=1$.

The angle formed by the module of the vector of state $\mathrm{P}(\psi)$ and the certainty degree axis $x$ is denominated by the inclination angle of the vector of state $\alpha_{\psi}$

The representation in the interlaced bilattice FOUR of the four superposed paraquantum logical states can be made through the set of complex numbers $C$, as shown below:

$$
\begin{gathered}
\psi_{(P Q) 1}=D c_{(p) t 1}+D c t_{(p) t 1} i \\
\psi_{(P Q) 2}=-D c_{(p) t 2}-D c t_{(p) t 2} i \\
\psi_{(P Q) 3}=-D c_{(p) f 1}+D c t_{(p) f 1} i \\
\psi_{(P Q) 4}=-D c_{(p) f 2}-D c t_{(p) f 2} i
\end{gathered}
$$

From Equation (1), we can also represent $\left(1-D c_{(p)}\right)=\alpha$ and $D c t_{(p)}=\beta$, then the four corresponding state vectors $\mathrm{P}(\psi)$ are

$$
\left|\psi_{(P Q) 1}\right\rangle=\left(1-\left|D c_{(p) t 1}\right|\right)|10\rangle+D c t_{(p) t 1}|11\rangle
$$

where $\left(1-\left|D c_{(p) t 1}\right|\right)^{2}+D c t_{(p) t 1}^{2}=1$

$$
\left\langle\psi_{(P Q) 2}\left|=\left(1-\left|D c_{(p) t 2}\right|\right)\right| 10\right\rangle-D c t_{(p) t 2}|00\rangle
$$

where $\left(1-\left|D c_{(p) t 2}\right|\right)^{2}+D c t_{(p) t 2}^{2}=1$

$$
\neg\left|\psi_{(P Q) 3}\right\rangle=\left(1-\left|D c_{(p) f 1}\right|\right)|01\rangle+D c t_{(p) f 1}|11\rangle
$$

where $\left(1-\left|D c_{(p) f 1}\right|\right)^{2}+D c t_{(p) f 1}^{2}=1$

$$
\neg\left\langle\psi_{(P Q) 4}\left|=\left(1-\left|D c_{(p) f_{2}}\right|\right)\right| 01\right\rangle-D c t_{(p) f_{2}}|00\rangle
$$

where $\left(1-\left|D c_{(p) f 2}\right|\right)^{2}+D c t_{(p) f 2}^{2}=1$

\subsubsection{Action of Logical Negation and of Logical Conflation for the Two Bernoulli Trials in the Interlaced Bilattice FOUR}

Descriptions of the actions of the operation of logical negation and the operation of logical conflation for two Bernoulli trials in simultaneous mode at interlaced bilattice FOUR are as follows. For the superposed paraquantum logical states from Equations (51) to (54), we have the following:

For $\psi_{(P Q)) 1}=\left(D c_{(p) t 1}, D c t_{(p) t 1}\right)$, the annotation is $\left[\mu_{(p) t 1}, \lambda_{(p) t 2}\right]$ or from Equations $((30)$ and $(31))$ is $[2 \sigma, \sqrt{\sigma}]$.

For $\psi_{(P Q) 2}=\left(D c_{(p) t 2}, D c t_{(p) t 2}\right)$, the annotation is $\left[\mu_{(p) t 2}, \lambda_{(p) t 1}\right]$ or from Equ- 
ations ((32) and (33)) is $[1-\sqrt{\sigma}, 1-2 \sigma]$.

For $\psi_{(P Q) 3}=\left(D c_{(p) f 1}, D c t_{(p) f 1}\right)$, the annotation is $\left[\mu_{(p) f 1}, \lambda_{(p) f 2}\right]$ or from Equations ((34) and (35)) is $[\sqrt{\sigma}, 2 \sigma]$.

For $\psi_{(P Q) 4}=\left(D c_{(p) f 2}, D c t_{(p) f 2}\right)$, the annotation is $\left[\mu_{(p) f 2}, \lambda_{(p) f 1}\right]$ or from Equations ((36) and (37)) is $[1-2 \sigma, 1-\sqrt{\sigma}]$.

From Equation (7), $\sigma=\sqrt{\sigma^{2}}=\sqrt{p-p^{2}}$; therefore, the variation of standard deviation $\sigma$ is $0.0 \leq \sigma \leq 0.5$, resulting in evidence degree

$\left(1-\frac{1}{\sqrt{2}}\right) \leq 2 \sigma \leq 1.0$ and evidence degree $0.0 \leq \sqrt{\sigma} \leq \frac{1}{\sqrt{2}}$. Since the logical negation operation from Equation (7) is $\neg\left[\mu_{(p)}, \lambda_{(p)}\right]=\left[\lambda_{(p) t}, \mu_{(p)}\right]$,

$$
\psi_{(P Q) 1}=\neg \psi_{(P Q) 3}
$$

and

$$
\begin{aligned}
& \psi_{(P Q) 3}=\neg \psi_{(P Q) 1} \\
& \psi_{(P Q) 2}=\neg \psi_{(P Q) 4}
\end{aligned}
$$

and

$$
\psi_{(P Q) 4}=\neg \psi_{(P Q) 2}
$$

Since the operation of logical conflation from Equation (18) is

$$
-\left[\mu_{(p)}, \lambda_{(p)}\right]=\left[1-\lambda_{(p) t}, 1-\mu_{(p)}\right],
$$

and

$$
\begin{aligned}
& \psi_{(P Q) 2}=-\psi_{(P Q) 1} \\
& \psi_{(P Q) 3}=-\psi_{(P Q) 4}
\end{aligned}
$$

and

$$
\psi_{(P Q) 4}=-\psi_{(P Q) 3}
$$

Figure 8 shows the $p$ PQL-Model associated at interlaced bilattice FOUR (represented by evidence degrees at probability $p$ obtained by Equations (30) to (33)) with four superposed paraquantum logical states $\psi_{\text {sup }}$ and four state vectors $\mathrm{P}(\psi)$ of unitary module with inclination angle $\alpha_{\psi}=\pi / 4$.

\section{Essays with $p \mathrm{PQL}-M o d e l$ Associated at Interlaced Bilattice FOUR}

With the $p$ PQL-Model associated at interlaced bilattice FOUR, a representation was built that enables good visualization of the superposed paraquantum logical states behavior.

The probability-valued trials show results that can be represented both numerically and graphically. 


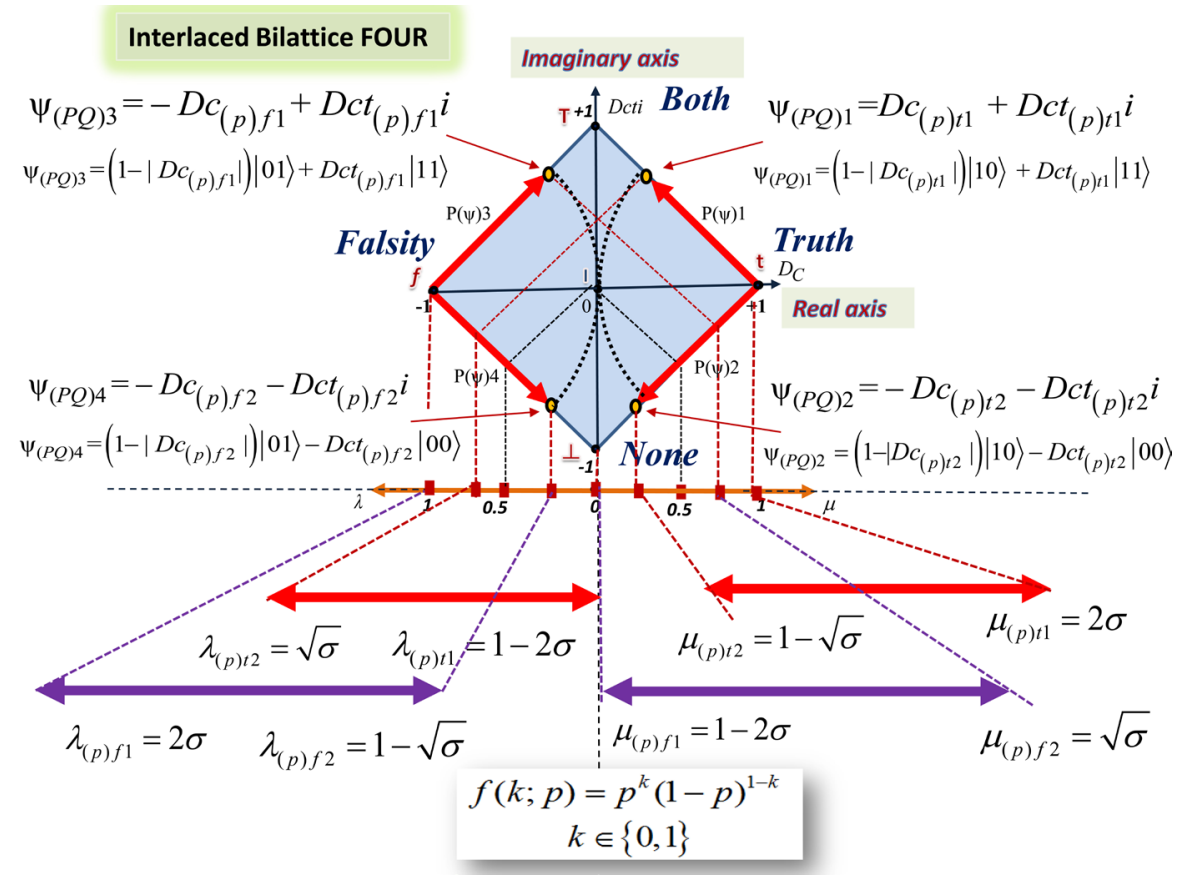

Figure 8. $p$ PQL-Model associated at interlaced bilattice FOUR with four state vectors $\mathrm{P}(\psi)$ of unitary module and superposed paraquantum logical states $\psi_{\text {sup. }}$.

\subsection{Results of Application of Probability Mass Function}

Application of the pmf of the Bernoulli distribution causes changes in values of the degrees of evidence; the appearance of trajectory of the superposed paraquantum logical states located at the arrowheads at the ends of the state vectors.

For example, Figure 9 shows the results for the application of evidence degrees at probability $p \cong 0.067$ to $p \cong 0.933$ of the module of state vector $\mathrm{P}(\psi)$ in interlaced bilattice FOUR. The error in establishing the unitary module for the state vector $\mathrm{P}(\psi)$ is small (maximum 6.3\%).

When $p \cong 0.067$ and $p \cong 0.933$, all paraquantum logical states are concentrated on the equidistant point of vertices of bilattice FOUR (Undefined logical state I). In this condition, the same features of a single trial Bernoulli with $50 \%$ probability appear and we have the following values:

The annotation of paraquantum logical state $\psi_{(P Q) 1}$ is $\left[\mu_{(p) t 1}, \lambda_{(p) t 2}\right]=\left[\frac{1}{2}, \frac{1}{2}\right]$ and, from Equation (51), the representation of paraquantum logical state in $C$ is $\psi_{(P Q) 1}=1+0 i$ and, from Equation (55), the paraquantum logical state is $\psi_{(P Q) 1}=|10\rangle$.

The annotation of paraquantum logical state $\psi_{(P Q) 2}$ is $\left[\mu_{(p) t 2}, \lambda_{(p) t 1}\right]=\left[\frac{1}{2}, \frac{1}{2}\right]$ and, from Equation (52), the representation of paraquantum logical state in $C$ is $\psi_{(P Q) 2}=1-0 i$ and, from Equation (56), the paraquantum logical state is $\psi_{(P Q) 2}=|10\rangle$.

The annotation of paraquantum logical state $\psi_{(P Q) 3}$ is 


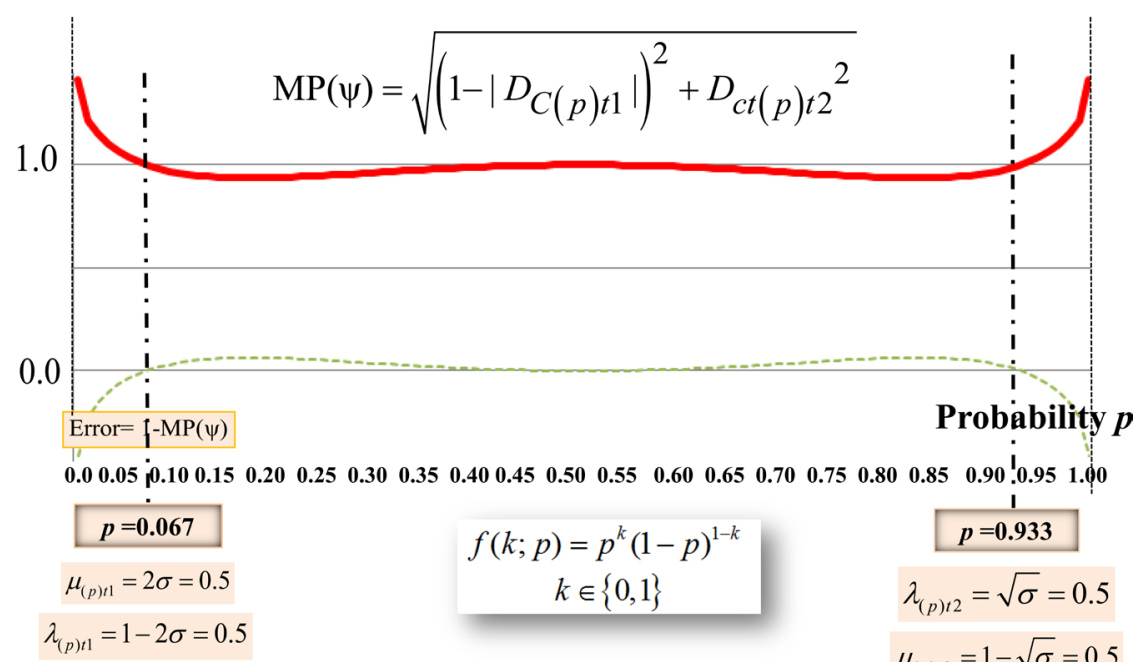

Figure 9. Results for evidence degrees at $p=0.067$ to $p=0.933$ of the module of state vector $\mathrm{P}(\psi)$ and error.

$\left[\mu_{(p) f 1}, \lambda_{(p) f 2}\right]=\left[\frac{1}{2}, \frac{1}{2}\right]$ and, from Equation (53), the representation of paraquantum logical state in $C$ is $\psi_{(P Q) 3}=-1+0 i$ and, from Equation (57), the paraquantum logical state is $\psi_{(P Q) 3}=|01\rangle$.

The annotation of paraquantum logical state $\psi_{(P Q) 4}$ is $\left[\mu_{(p) f 2}, \lambda_{(p) f 1}\right]=\left[\frac{1}{2}, \frac{1}{2}\right]$ and, from Equation (54), the representation of paraquantum logical state in $C$ is $\psi_{(P Q) 4}=-1-0 i$ and, from Equation (58), the paraquantum logical state is $\psi_{(P Q) 4}=|01\rangle$.

From the analysis in this condition, only two logical states remained, $\psi_{(P Q) 1}=\psi_{(P Q) 2}=|10\rangle$ and $\psi_{(P Q) 3}=\psi_{(P Q) 4}=|01\rangle$.

\subsection{Results for Entangled Bell States}

With the $p$ PQL-Model associated at interlaced bilattice FOUR, we can obtain values for entangled Bell states [30] [34]. The entangled Bell states are configured at $p=0.5$ with the following values:

The annotation of paraquantum logical state $\psi_{(P Q) 1}$ is $\left[\mu_{(p) t 1}, \lambda_{(p) t 2}\right]=\left[1, \frac{1}{\sqrt{2}}\right]$ and, from Equation (51), the representation of paraquantum logical state in $C$ is $\psi_{(P Q) 1}=\left(1-\frac{1}{\sqrt{2}}\right)+\frac{1}{\sqrt{2}} i$ and, from Equation (55), the paraquantum logical state is $\psi_{(P Q) 1}=\left(\frac{1}{\sqrt{2}}\right)|10\rangle+\frac{1}{\sqrt{2}}|11\rangle$.

The annotation of paraquantum logical state $\psi_{(P Q) 2}$ is $\left[\mu_{(p) t 2}, \lambda_{(p) t 1}\right]=\left[\left(1-\frac{1}{\sqrt{2}}\right), 0\right]$ and, from Equation (52), the representation of 
paraquantum logical state in $C$ is $\psi_{(P Q) 2}=\left(1-\frac{1}{\sqrt{2}}\right)-\frac{1}{\sqrt{2}} i$ and, from Equation (56), the paraquantum logical state is $\psi_{(P Q) 2}=\left(\frac{1}{\sqrt{2}}\right)|10\rangle-\frac{1}{\sqrt{2}}|00\rangle$.

The annotation of paraquantum logical state $\psi_{(P Q) 3}$ is $\left[\mu_{(p) f 1}, \lambda_{(p) f 2}\right]=\left[\frac{1}{\sqrt{2}}, 1\right]$ and, from Equation (53), the representation of paraquantum logical state in $C$ is $\psi_{(P Q) 3}=-\left(1-\frac{1}{\sqrt{2}}\right)+\frac{1}{\sqrt{2}} i$ and, from Equation (57), the paraquantum logical state is $\psi_{(P Q) 3}=\left(\frac{1}{\sqrt{2}}\right)|01\rangle+\frac{1}{\sqrt{2}}|11\rangle$.

The annotation of paraquantum logical state $\psi_{(P Q) 4}$ is $\left[\mu_{(p) f 2}-\lambda_{(p) f 1}\right]=\left[0,\left(1-\frac{1}{\sqrt{2}}\right)\right]$ and, from Equation (54), the representation of paraquantum logical state in $C$ is $\psi_{(P Q) 4}=-\left(1-\frac{1}{\sqrt{2}}\right)-\frac{1}{\sqrt{2}} i$ and, from Equation (58), the paraquantum logical state is $\psi_{(P Q) 4}=\left(\frac{1}{\sqrt{2}}\right)|01\rangle-\frac{1}{\sqrt{2}}|00\rangle$.

The range of variation that takes the superposed paraquantum states from the Bell logical state to uncertainty is $\Delta p=0.933-0.067=0.866$. Figure 10 shows the results of the variation in the degree of contradiction and the complement of the degrees of certainty over a range of probability variations in simultaneous mode: in one direction to the False logical state $\Delta_{p f}=0.5 \leftrightarrow 0.067$ and in the

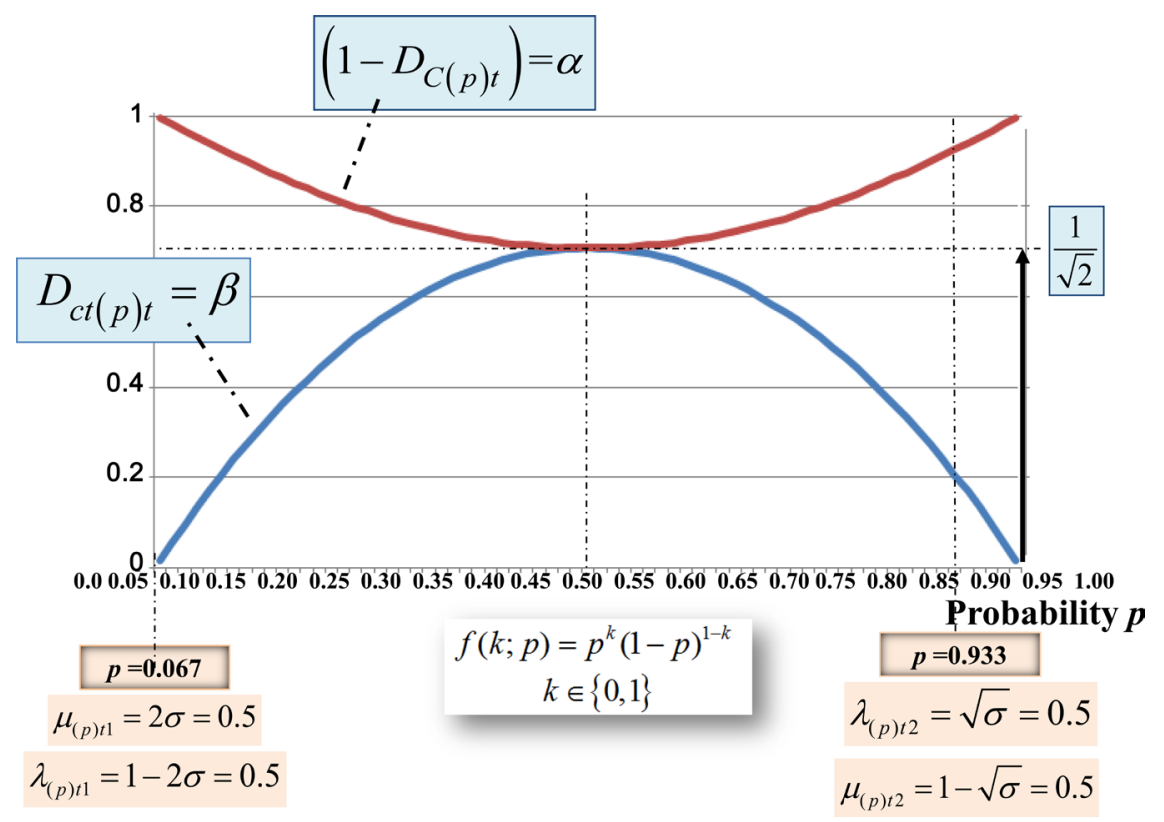

Figure 10. Results of the variation in the degree of contradiction and the complement of the degrees of certainty over a range of probability variations in simultaneous mode from $\Delta_{p f}=0.5 \leftrightarrow 0.067$ and $\Delta_{p t}=0.5 \leftrightarrow 0.933$. 
other direction to the True logical state $\Delta_{p t}=0.5 \leftrightarrow 0.933$.

\subsection{Application of Cumulative Distribution Function (CDF)}

The cumulative distribution function (CDF) can be used to establish stationary logic states represented in the interlaced bilattice FOUR. In this mode of representation, the stationary logical states will be located at points where the error is minimal. For example, we can choose the two states where the error is zero and in the discrete analysis a quantum leap would occur.

For continuum analysis between the two intervals, the pmf of the Bernoulli distribution would be used.

Figure 11 shows the application of $\mathrm{CDF}$ in the intervals:

$p=0 \rightarrow p=0.067$ and $p=0.933 \rightarrow p=1.0$; here the collapse of the wave function occurs; $p=0.067 \rightarrow p=0.45$ and $p=0.55 \rightarrow p=0.933$; here the steady state of Bell occurs; $p=0.433 \rightarrow p=0.567$, which is the undefinite logical state; in this interval, the state is governed by pmf.

The result is the vector state module of practically unitary, $\operatorname{MP}(\psi)=1$.

\subsection{Comments and Discussion}

In this work, the $p$ PQL-Model associated at interlaced bilattice FOUR was presented in two ways.

The first mode was for the single Bernoulli trials process [28] [32] [35], where the experiment consists of just one flip of a coin. In this case, the outcome is either heads $(\mathrm{H})$ or tails $(\mathrm{T})$, and the sample space is represented by $\Omega=\{\mathrm{H} \mathrm{T}\}$. This means that after the final measurement, the result can only point to a logical state represented by $\{\mathrm{H}\}$ or $\{\mathrm{T}\}$.

The second mode was for the two Bernoulli trials process, where the experiment consists of the flipping of two coins simultaneously: on launch the $\sigma$-algebra $\mathrm{F}=2 \Omega$ contains $2^{2}=4$ events, namely, $\{\mathrm{H} \mathrm{H}\}$ heads, heads; $\{\mathrm{T} \mathrm{T}\}$ tails,

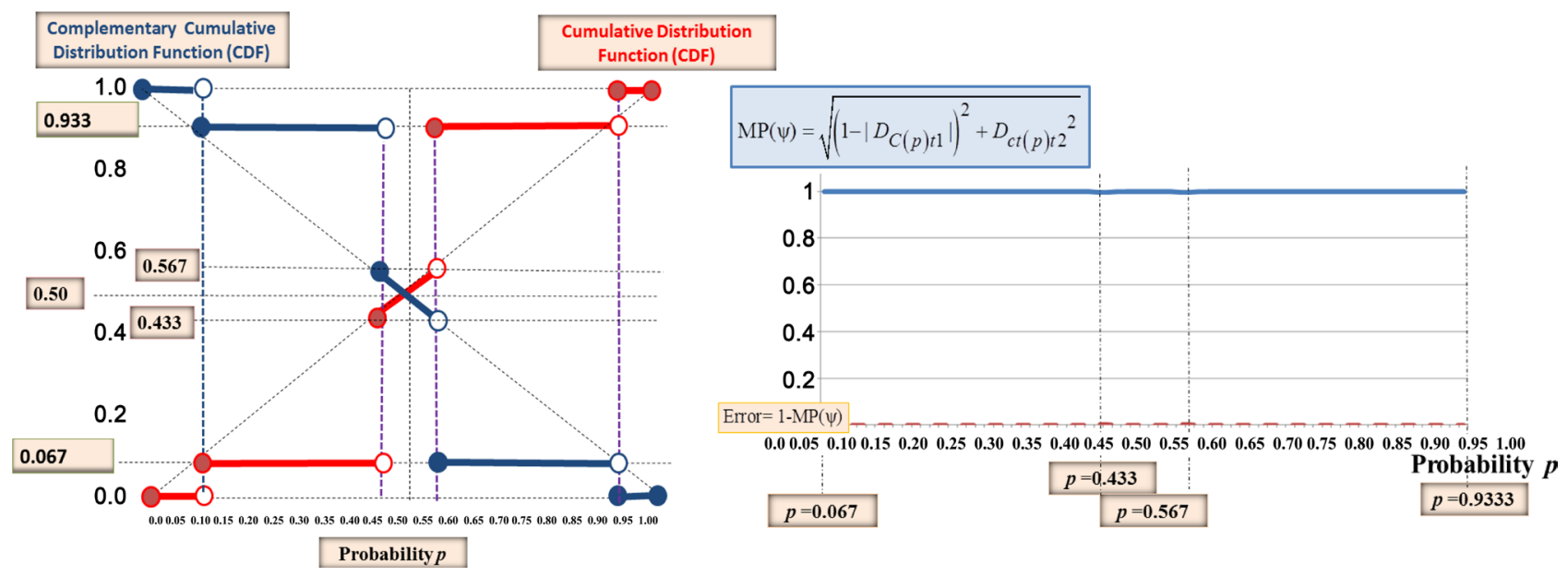

Figure 11. Application of cumulative distribution function (CDF) in the probability mass function (pmf) of the Bernoulli distribution; (a) CDF and complementary cumulative distribution function (CCDF); (b) graphic results for the module of state vector $\mathrm{P}(\psi)$ and error. 


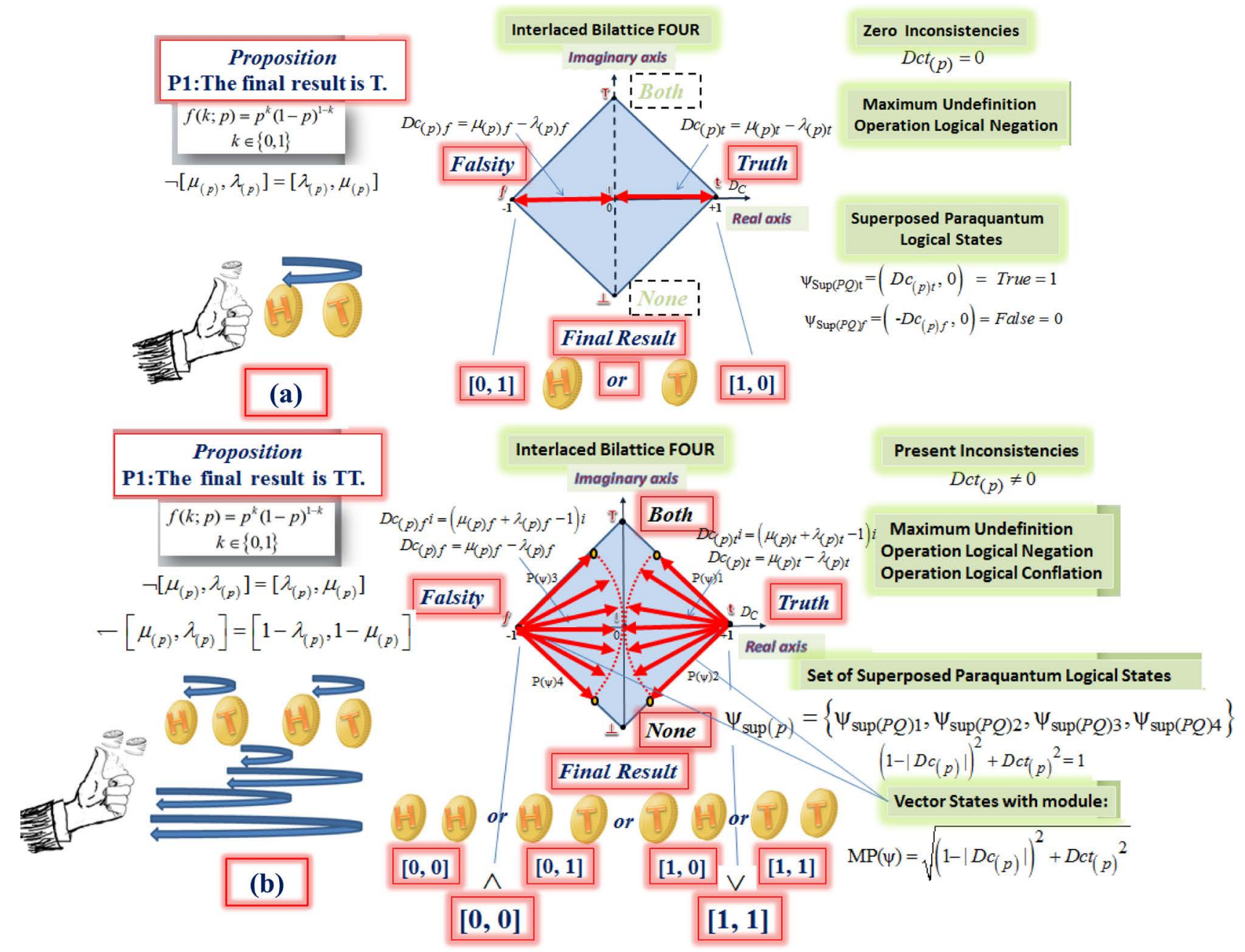

Figure 12. Two ways of PQL analysis, logical states and quantum effects. (a) Single Bernoulli Trial on the interlaced bilattice FOUR. (b) Two Bernoulli trials in simultaneous mode at interlaced bilattice FOUR.

tails; $\{\mathrm{H} \mathrm{T}\}$ heads, tails; and $\{\mathrm{T} \mathrm{H}\}$ tails, heads. This means that after the final measurement, the result will only point out a logical state represented by $\{\mathrm{H} \mathrm{H}\}$ or $\{\mathrm{T} \mathrm{T}\}$ or $\{\mathrm{H} \mathrm{T}\}$ or $\{\mathrm{T} \mathrm{H}\}$.

Figure 12 shows the two analyses in PQL, the logical states, the consequences and equations of quantum effects.

For the paraconsistent logic with annotation of two-values, PAL2v, the resulting logical state for both modes after the final measurement will depend on the proposition used in the analysis.

\subsubsection{Before Final Measurement}

Before final measurement the Bernoulli trials process is modelled by the $p$ PQL-Model associated at interlaced Bilattice FOUR. As seen in this study, in the $p$ PQL-Model, the logical conflation was null when in the single Bernoulli trial study; however, it appears and produces the indistinguishability in case of two Bernoulli trials in simultaneous mode. For this second mode, before measuring the pmf of the Bernoulli distribution, it produces the probability variations $p$ and 
causes variations in degrees of favorable evidence $\mu_{(p)}$ and unfavorable evidence $\lambda_{(p)}$. Due to these variations, the state vectors $\mathrm{P}(\psi)$ in the interlaced bilattice FOUR move and make the trajectory of the superposed paraquantum logical states $\psi_{\text {sup }}$.

The logical conflation and logical negation in the interlaced bilattice FOUR produce the entanglement and superposition in both states. The superposition happens horizontally between False $(f)$ and True $(t)$, and in simultaneous mode, the superposition happens vertically with the exchange of vectors of states $\mathrm{P}(\psi)$ through the complex conjugate of the paraquantum logical states $\psi_{\text {pql }}$.

With this reasoning, we can say that in the interlaced bilattice FOUR, the undefinition by superposition of horizontal states is represented by logical negation (Equation (7)). In contrast, the change of the state vectors, through complex conjugate of the logical state causes undefinition, represented in the interlaced bilattice FOUR by knowledge negation (conflation) (Equation (18)). Therefore, the logical operations conflation and negation are responsible for the effects of the quantum phenomena (entanglement and superposition) that are observed in the bilattice FOUR.

\subsubsection{Final Result after Measurement}

The final result after measuring identifies the consequences of superposition and entanglement of logical states in the interlaced bilattice FOUR.

Entanglement occurs due to ordering of the interlaced bilattice FOUR and to satisfy one of two classic logical states (True or False).

The knowledge negation (conflation) and logical negation mean that before measurement, we cannot distinguish the coins and the logical state of each of them. After the final measurement, the state vectors $\mathrm{P}(\psi)$ and the wave function collapse and the result will be one of the four logical states represented at the vertices of interlaced bilattice FOUR obeying the ordering of knowledge $\leq_{k}$ and truth $\leq_{t}$ of the bilattice. Meet $(\Lambda)$ and join $(V)$ operators under $\leq_{t}$ are then used such that

For final results, False $f$ or Paracomplete $\perp$, as $f \leq_{t} \perp \leq_{t} t$, then the meet operator $(\Lambda)$ is applied:

o Case results in False $f$, then the final result will be False $f$

o Case results in Paracomplete $\perp$, then the final result will be false $f$

For final results True $t$ or Inconsistent $\mathrm{T}$, as $f \leq_{\mathrm{t}} \mathrm{T} \leq_{\mathrm{t}} t$, then the Join operator $(V)$ is applied:

o Case results in True $t$, then the final result will be True $t$

o Case results in Inconsistent $\mathrm{T}$, then the final result will be True $t$

Finally, from the Equation (11c) $f \otimes t=\perp$ and (11a) $\perp \wedge T=f$, the application of the meet operator $(\wedge)$ will result in the final outcome False $(f)$ for $[00]$ and $\left[\begin{array}{ll}0 & 1\end{array}\right]$ :

$$
[0,0] \wedge[0,1]=[0,0] \rightarrow[\mathrm{T}, \mathrm{T}] \wedge[\mathrm{T}, \mathrm{H}]=f
$$

From the Equation (11d), $f \oplus t=\mathrm{T}$, and (11b), $\perp \vee T=t$, the application of 
the join operator $(V)$ will result in the final outcome true $(t)$ for $\left[\begin{array}{ll}1 & 1\end{array}\right]$ or $\left[\begin{array}{ll}1 & 0\end{array}\right]$ :

$$
[1,1] \vee[1,0]=[1,1] \rightarrow[\mathrm{H}, \mathrm{H}] \vee[\mathrm{H}, \mathrm{T}]=t
$$

\subsubsection{Example: Before and after Measurement}

Before measurement, the analyses on interlaced bilattice FOUR have a proposition $P$, which can be formed, for example, by launching two coins simultaneously:

$P 1$ : There is the probability $p$ of the occurrence of two $\mathrm{H}$.

On representation of the bilattice FOUR the result of two $\mathrm{H}$ is the Inconsistent Logical State T.

In this condition, the evidence degrees of the annotation $\left[\mu_{(p)}, \lambda_{(p)}\right]$ are transformed in maximum (1) or minimum (0) values

$\left[\mu_{(p) \max } \vee \mu_{(p) \min }, \lambda_{(p) \max } \vee \lambda_{(p) \min }\right]$ :

The True logical state $(t)$ after the measurement is [1 1 l] symbolized in the bilattice FOUR for T.

The Inconsistent logical state $(T)$ after the measurement is [1 0$]$ symbolized in the bilattice FOUR for $t$.

The Paracomplete logical state $(\perp)$ after the measurement is [0 1 1] symbolized in the bilattice FOUR for $f$.

The False logical state $(f)$ after the measurement is $[00]$ symbolized in the bilattice FOUR for $\perp$.

$P 2$ : There is the probability $p$ of the occurrence of two T.

On representation of the bilattice FOUR, the result of two T is the Paracomplete logical state $\perp$. In this condition, we have the following:

The True logical state $(t)$ after the measurement is $\left[\begin{array}{ll}0 & 0\end{array}\right]$ symbolized in the bilattice FOUR for $\perp$.

The Inconsistent logical state $(T)$ after the measurement is [1 0$]$ symbolized in the bilattice FOUR for $t$.

The Paracomplete logical state $(\perp)$ after the measurement is $\left[\begin{array}{ll}0 & 1\end{array}\right]$ symbolized in the bilattice FOUR for $f$.

The False logical state $(f)$ after the measurement is [ $\left[\begin{array}{ll}1 & 1\end{array}\right]$ symbolized in the bilattice FOUR for $T$.

$P 3$ : There is the probability $p$ of the occurrence of one $\mathrm{H}$ and one $\mathrm{T}$.

On representation of the bilattice FOUR, the result of one $\mathrm{H}$ and one $\mathrm{T}$ is the logical state True $t$. In this condition, we have the following:

The True logical state $(t)$ after the measurement is $\left[\begin{array}{ll}1 & 0\end{array}\right]$ symbolized in the bilattice FOUR for $t$.

The Inconsistent logical state $(T)$ after the measurement is [1 11$]$ symbolized in the bilattice FOUR for $T$.

The Paracomplete logical state $(\perp)$ after the measurement is [0 0$]$ symbolized in the FOUR bilattice for $\perp$.

The False logical state $(f)$ after the measurement is [0 1$]$ symbolized in the bilattice FOUR for $f$.

P4: There is the probability $p$ of the occurrence of one $\mathrm{T}$ and one $\mathrm{H}$. 
On representation of the bilattice FOUR, the result of one $\mathrm{T}$ and one $\mathrm{H}$ is the False logical state $f$. In this condition, we have the following:

The True logical state $(t)$ after the measurement is [0 1$]$ symbolized in the bilattice FOUR for $f$.

The Inconsistent logical state $(T)$ after the measurement is [ 11 1] symbolized in the bilattice FOUR for $T$.

The Paracomplete logical state $(\perp)$ after the measurement is $\left[\begin{array}{ll}0 & 0\end{array}\right]$ symbolized in the bilattice FOUR for $\perp$.

The False logical state $(f)$ after the measurement is [ 10$]$ symbolized in the bilattice FOUR for $t$.

\subsubsection{Correlation with EPR Paradox}

With the $p$ PQL-Model associated at interlaced bilattice FOUR, we can study certain concepts of correlation that are similar to the EPR paradox.

As it was seen, the mapping of degrees of probabilistic evidence $(p)$ in the interlaced bilattice FOUR resulted in each of its four vertices represented by an annotation, or a pair of degrees of probabilistic evidence with their extreme values, where

$$
\begin{aligned}
& \mu_{(p)}=1 \text { and } \lambda_{(p)}=1 \quad \text { Inconsistent logical state }[1,1]=\mathrm{T} \\
& \mu_{(p)}=1 \text { and } \lambda_{(p)}=0 \quad \text { True logical state }[1,0]=t \\
& \mu_{(p)}=0 \text { and } \lambda_{(p)}=0 \quad \text { Paracomplete logical state }[0,0]=\perp \\
& \mu_{(p)}=0 \text { and } \lambda_{(p)}=1 \quad \text { False logical state }[0,1]=f
\end{aligned}
$$

We can consider that, in the Bernoulli experiment of launching two coins simultaneously, after entanglement, agent A (Alice) chooses one of them at random. Then, leaving the other coin with agent $\mathrm{B}$ (Bob), agent A travels to a distant region.

The proposition previously chosen is $P 1$ : There is the probability $p$ of the occurrence $\mathrm{HH}$. When distant from each other, if agent A (or agent B) does a measurement, then only the individual state $0(\mathrm{~T})$ or $1(\mathrm{H})$ for one coin will be known.

The final logical state of the probabilistic paraquantum model, represented at the vertices of bilattice FOUR by two extreme values of the pair $[\mu, \lambda]$ will be known only by agents A and B when the measurements of logical states of the two coins are made. However, irrespective of the distance, when we consider that agent A (or B) is in the position of the $\mu$-degree of favorable evidence probabilistic, when $s /$ he does the measurement of the coin, s/he will instantly know the resultant final state. For example, considering that the pair $[\mu, \lambda]=[\mathrm{A}, \mathrm{B}]$ :

If agent $\mathrm{A}$ does the measurement and it results in $\mathrm{H}$, the pair of extreme values will be $[1 \mathrm{~B}]$. In this condition, if agent $\mathrm{B}$ does a measurement and it results in $\mathrm{T}$, the pair of extreme values will be [1 0], resulting in the final logical state true $(t)$. However, if agent $\mathrm{B}$ does a measurement resulting in $\mathrm{H}$, the pair of extreme values will be [1 1]; that is, an Inconsistent logical state $T$ and the connective $V$ of bilattice FOUR (join operator $\vee$ ) is applied and the final logical state will be true $(t)$. 
If agent $\mathrm{A}$ does the measurement and it results in $\mathrm{T}$, then the pair of extreme values will be $[0 \mathrm{~B}]$. In this condition, if agent $\mathrm{B}$ does a measurement and it results in $\mathrm{H}$, the pair of extreme values will be [0 1], resulting in the final logical state false $(f)$. However, if agent B does a measurement resulting in T, the pair of extreme values will be [0 0]; that is, a Paracomplete logical state $\perp$ and the connective $\wedge$ of bilattice FOUR (meet operator $\wedge$ ) is applied and the final logical state will be false $(f)$.

The position of agent $\mathrm{B}$ as $\lambda$, degree of unfavorable evidence probabilistic, does not allow that he knows the result of the final logical state when he does the coin measurement. Therefore, in communication systems using the paraquantum logical model, we must establish which of the agents is initially positioned as the $\mu$-probabilistic degree of favorable evidence. Another way is that the choice can be random, where the two agents know that there is a mark in one of the two coins.

The coin with the mark has the condition to position itself as $\mu$-probabilistic degree of favorable evidence. Due the indistinguishability of the coins in the interlacing of the bilattice FOUR when the separation of the entangled coins is made, none of the agents know if your chosen coin is the one that has the mark. This condition will only be known when the agent does the measurement.

\section{Conclusion Remarks}

In this paper, we presented a logical model that combines non-relativistic quantum mechanics with probabilistic theory of the Bernoulli distribution, bilattices theory, and foundations of paraconsistent logic. With the union of these theories, it was possible to build a logical and probabilistic model that works through the foundations of paraconsistent annotated logic with the ability to simulate phenomena found in quantum physics. With the inclusion of paraconsistent logic in physical science, this model was named the probabilistic paraquantum logical model ( $p$ PQL-Model). In this work, the $p$ PQL-Model proved to be innovative because it uses projections in an interlaced bilattice FOUR, which has properties that enhance studies in quantum mathematics and the visualization of quantum phenomena. In this model, the operation of logical conflation was introduced as a new and important concept to the appropriateness of quantum logic with the interlaced bilattice FOUR. In the PQL logic, the logical conflation has fundamental importance due to its property of indistinguishability, which is characteristic of the entangled logical States. Using the concepts of PQL, we can verify that the operation of logical conflation is responsible for providing suitable modeling at various phenomena of QM. The equations used in the model are of medium complexity and are ideal for creating algorithmic structures considered essential for efficient quantum computing with low computational cost. The results in this work indicate that the pPQL-Model, based in non-classical paraconsistent logic and the theory of interlaced bilattices, ushers in a new field of research. For research in quantum physics, the algorithmic computational 
structure of the $p \mathrm{PQL}-$ Model will serve for the construction of new quantum logic gates, quantum algorithms, and efficient circuits applied in quantum analysis systems.

\section{References}

[1] Landau, L.D. and Lifshitz, E.M. (2003) Quantum Mechanics (Non-Relativistic Theory). Butterworth Heinemann. Chapter 1.

[2] Feynman, R.P. (1965) The Feynman Lectures on Physics. Volume III, Chapter 1. Volume I, Chapters 30 and 38.

[3] Feynman, R.P. (1948) Space-Time Approach to Non-Relativistic Quantum Mechanics. Reviews of Modern Physics, 20, 367. https://doi.org/10.1103/RevModPhys.20.367

[4] Feynman, R.P. and Hibbs, R.H. (1965) Quantum Mechanics and Path Integrals. McGraw-Hill.

[5] Birkhoff, G. and von Neumann, J. (1936) The Logic of Quantum Mechanics. Annals of Mathematics, 37, 823-843. https://doi.org/10.2307/1968621

[6] Birkhoff, G. (1967) Lattice Theory. American Mathematical Society, Providence.

[7] Piron, C. (1976) Foundations of Quantum Physics. W.A. Benjamin, Inc. https://doi.org/10.1007/978-94-010-1440-3_7

[8] Machey, G.M. (1963) The Mathematical Foundations of Quantum Mechanics. W. A. Bejamin, New York.

[9] Engesser, K., Gabbay, D.M. and Lehmann, D.A. (2007) New Approach to Quantum Logic (Studies in Logic Book 8). College Publications, $200 \mathrm{p}$.

[10] Ekert, D.A. and Lupacchini, R. (2000) Machines, Logic and Quantum Physics. Bulletin of Symbolic Logic, 3, 265-283.

[11] Dishkant, H. (1972) Semantics of the Minimal Logic of Quantum Mechanics. Studia Logica, 30, 17-29. https://doi.org/10.1007/BF02120818

[12] Redei, M. (1998) Quantum Logic in Algebraic Approach. Kluwer Academic Publishers. https://doi.org/10.1007/978-94-015-9026-6

[13] Dalla Chiara, M.L. and Giuntini, R. (1989) Paraconsistent Quantum Logics. Foundations of Physics, 19, 891-904. https://doi.org/10.1007/BF01889304

[14] Dalla Chiara, M.L. and Giuntini, R. (1994) Unsharp Quantum Logics. Foundations of Physics, 24, 1161-1177. https://doi.org/10.1007/BF02057862

[15] Dalla Chiara, M., Giuntini, R. and Greechie, R. (2004) Reasoning in Quantum Theory. Kluwer Academic Publishers, Dordrecht. https://doi.org/10.1007/978-94-017-0526-4

[16] Abe, J.M., Nakamatsu, K., Akama, S. and Da Silva Filho, J.I. (2018) The Importance of Paraconsistency and Paracompleteness in Intelligent Systems. In: Czarnowski, I., Howlett, R. and Jain, L., Eds., Intelligent Decision Technologies 2017. IDT 2017. Smart Innovation, Systems and Technologies, Vol. 73. Springer, Cham. https://doi.org/10.1007/978-3-319-59424-8_18

[17] Da Costa, N.C.A. and de Ronde, C. (2013) The Paraconsistent Logic of Quantum Superpositions. Foundations of Physics, 43, 845. https://doi.org/10.1007/s10701-013-9721-9

[18] Da Silva Filho, J.I., Nunes, V.C., Garcia, D.V.N., Mario, M.C., Giordano, F., Abe, J.M. Pacheco, M.T.T. and Silveira Jr., L. (2016) Paraconsistent Analysis Network Applied in the Treatment of Raman Spectroscopy Data to Support Medical Diagno- 
sis of Skin Cancer. Reviews of Modern Physics, 54, 1-15. https://doi.org/10.1007/s11517-016-1471-3

[19] Da Costa, N.C.A. and Marconi, D. (1989) An Overview of Paraconsistent Logic in the 80's. The Journal of Non-Classical Logic, 6, 5-31.

[20] Subrahmanian, V.S. (1987) On the Semantics of Quantitative Logic Programs. Proc. 4th. IEEE Symposium on Logic Programming, Computer Society Press, Washington DC.

[21] Da Silva Fiho, J.I., Lambert-Torres, G. and Abe, J.M. (2010) Uncertainty Treatment Using Paraconsistent Logic-Introducing Paraconsistent Artificial Neural Networks. In: Volume 211-Frontiers in Artificial Intelligence and Applications, IOS Press, Amsterdam, 328 p.

[22] Da Silva Filho, J.I. (2016) Undulatory Theory with Paraconsistent Logic (Part I): Quantum Logical Model with Two Wave Functions. Journal of Quantum Information Science, 6, 143-180. https://doi.org/10.4236/jqis.2016.63012

[23] Da Silva Filho, J.I. (2016) Undulatory Theory with Paraconsistent Logic (Part II): Schrödinger Equation and Probability Representation. Journal of Quantum Information Science, 6, 181-213. https://doi.org/10.4236/jqis.2016.63013

[24] Belnap, N. (1977) How a Computer Should Think. In: Ryle, G., Ed., Contemporary Aspects of Philosophy. Oriel Press, Stocksfield, 30-55.

[25] Belnap, N. (1977) A Useful Four-Valued Logic. In: Dunn, J.M. and Epstein, G., Eds., Modern Uses of Multiple-Valued Logic, Reidel, Dordrecht, 8-37. https://doi.org/10.1007/978-94-010-1161-7_2

[26] Davey, B.A. and Priestley, H.A. (2005) Introduction to Lattices and Order. Cambridge University Press, Cambridge.

[27] Wille, R. (1992) Concept Lattices and Conceptual Knowledge Systems. Computers \& Mathematics with Applications, 23, 493-515. https://doi.org/10.1016/0898-1221(92)90120-7

[28] Nedelman, J. and Wallenius, T. (1986) Bernoulli Trials, Poison Trials, Surprising Variances, and Jensens's Inequality. The American Statistician, 40, 286-289.

[29] Samuels, S.M. (1965) On the Number of Successes in Independent Trials. The Annals of Mathematical Statistics, 36, 1272-1278. https://doi.org/10.1214/aoms/1177699998

[30] Nielsen M.A. and Chuang, I.L. (2000) Quantum Computation and Quantum Information. Cambridge University Press.

[31] Rédei, M. (1989) Quantum Conditional Probabilities Are Not Probabilities of Quantum Conditional. Physics Letters A, 139, 287-290. https://doi.org/10.1016/0375-9601(89)90454-4

[32] Grinstead, C.M. and Snell, J.L. (1997) Introduction to Probability. Providence, RI, American Mathematical Society.

[33] Einstein, A., Podolsky, B. and Rosen, N. (1935) Can Quantum Mechanical Description of Physical Reality Be Considered Complete? Physical Review, 47, 777-780. https://doi.org/10.1103/PhysRev.47.777

[34] Ginsberg, M.L. (1988) Multivalued Logics: A Uniform Approach to Inference in Artificial Intelligence. Computational Intelligence, 4, 265-316. https://doi.org/10.1111/j.1467-8640.1988.tb00280.x

[35] Horodecki, M.P. and Horodecki, R. (2001) Mixed-State Entanglement and Quantum Communication. Quantum Information, Springer Tracts in Modern Physics, 173, 151-195. https://doi.org/10.1007/3-540-44678-8_5 
Submit or recommend next manuscript to SCIRP and we will provide best service for you:

Accepting pre-submission inquiries through Email, Facebook, LinkedIn, Twitter, etc. A wide selection of journals (inclusive of 9 subjects, more than 200 journals)

Providing 24-hour high-quality service

User-friendly online submission system

Fair and swift peer-review system

Efficient typesetting and proofreading procedure

Display of the result of downloads and visits, as well as the number of cited articles Maximum dissemination of your research work

Submit your manuscript at: http://papersubmission.scirp.org/

Or contact jqis@scirp.org 